\title{
Inclusive and exclusive observables from dipoles in high energy collisions ${ }^{1}$
}

\author{
Christoffer Flensburg, ${ }^{a}$ Gösta Gustafson ${ }^{a}$ and Leif Lönnblad ${ }^{a, b}$ \\ ${ }^{a}$ Dept. of Astronomy and Theoretical Physics, Lund University, \\ Sölvegatan 14A, Lund, Sweden \\ ${ }^{b}$ CERN Theory Unit, \\ 1211 Geneva 23, Switzerland \\ E-mail: christoffer.flensburg@thep.lu.se, gosta@thep.lu.se, \\ leif.lonnblad@thep.lu.se
}

ABSTRACT: We present a new model for simulating exclusive final states in minimum-bias collisions between hadrons.

In a series of papers we have developed a Monte Carlo model based on Mueller's dipole picture of BFKL-evolution, supplemented with non-leading corrections, which has shown to be very successful in describing inclusive and semi-inclusive observables in hadron collisions. In this paper we present a further extension of this model to also describe exclusive final states. This is a highly non-trivial extension, and we have encountered many details that influence the description, and for which no guidance from perturbative QCD could be found. Hence we have had to make many choices based on semi-classical and phenomenological arguments.

The end result is a new event generator called DIPSY which can be used to simulate complete minimum-bias non-diffractive hadronic collision events. Although the description of data from the Tevatron and LHC is not quite as good as for PYTHIA 8, the most advanced of the general purpose event generator programs for these processes, our results are clearly competitive, and can be expected to improve with careful tuning. In addition, as our model is very different from conventional multiple scattering scenaria, the DIPSY program can be used to gain deeper insight in the soft and semi-hard processes involved both in hadronic and heavy ion collisions.

KeYwords: QCD Phenomenology, Phenomenological Models

ARXiv EPRINT: 1103.4321

\footnotetext{
${ }^{1}$ Work supported in parts by the EU Marie Curie RTN MCnet (MRTN-CT-2006-035606), and the Swedish research council (contracts 621-2008-4252 and 621-2009-4076).
} 


\section{Contents}

1 Introduction 1

2 The Lund dipole cascade model for inclusive cross sections 3

2.1 Mueller's cascade model and the eikonal formalism 3

2.2 The Lund dipole cascade model 5

2.2.1 Beyond LL BFKL evolution $\quad 5$

$\begin{array}{ll}2.2 .2 \text { Non-linear effects and saturation } & 6\end{array}$

$\begin{array}{ll}2.2 .3 & \text { Confinement effects }\end{array}$

$\begin{array}{lll}2.3 & \text { Initial dipole configurations } & 7\end{array}$

$\begin{array}{lll}3 & \text { From inclusive to exclusive observables } & 8\end{array}$

3.1 The chain of $k_{\perp}$-changing gluons 8

$\begin{array}{ll}3.2 & \text { Reabsorption of virtual emissions } \\ 3.3 & \text { Giving }\end{array}$

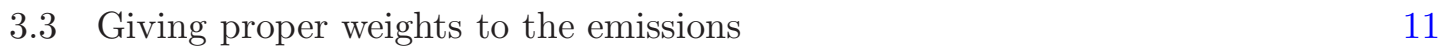

3.4 Going from transverse coordinate space to momentum space 12

$\begin{array}{ll}3.5 & \text { Final state radiation and hadronization } \\ \end{array}$

4 Generating the exclusive final states $\quad 14$

$\begin{array}{lll}4.1 & \text { Selecting the interactions } & 15\end{array}$

$\begin{array}{lll}4.2 & \text { Identifying the backbone gluons } & 15\end{array}$

$\begin{array}{lll}4.3 & \text { Reweighting outer } q_{\perp} \text { maxima } & 16\end{array}$

$\begin{array}{lll}4.4 & \text { FSR matching and ordering } & 16\end{array}$

$\begin{array}{lll}4.5 & \text { Colour flow } & 17\end{array}$

$\begin{array}{lll}4.6 & \text { Higher order corrections } & 17\end{array}$

$\begin{array}{lll}\text { 4.6.1 Coherence as relaxed ordering } & 17\end{array}$

$\begin{array}{lll}\text { 4.6.2 Saturation effects } & 18\end{array}$

5 Self-consistency and tuning $\quad 18$

$\begin{array}{ll}5.1 \text { Achieving frame independence } & 19\end{array}$

5.2 Tuning to experimental data 22

$\begin{array}{ll}5.3 & \text { Comparison with experiments } \\ \end{array}$

6 Conclusions $\quad 28$

$\begin{array}{lll}6.1 \text { Outlook } & 30\end{array}$

$\begin{array}{lll}\text { A } & q_{\perp} \text { max reweighting in DIPSY } & 31\end{array}$

B Absorbed partons and ordering 33

B.1 Coherence 33

$\begin{array}{lll}\text { B.2 Ordering in the interaction } & 35\end{array}$ 
$\begin{array}{ll}\text { C Saturation effects } & \mathbf{3 7}\end{array}$

$\begin{array}{lll}\text { C.1 Multiple interaction } & 37\end{array}$

$\begin{array}{lll}\text { C.2 The swing } & 38\end{array}$

$\begin{array}{lll}\text { C.3 Colour flow in saturated cascades } & 39\end{array}$

\section{Introduction}

In high energy collisions the high density of gluons at small $x$ imply that typical events contain several hard partonic subcollisions, and that saturation effects become important. A dynamical model based on BFKL evolution $[1,2]$ for small $x$ and including saturation effects, has been presented in a series of papers [3-7]. It is based on Mueller's dipole cascade model [8-10], which is a formulation of the leading logarithmic (LL) BFKL evolution approximation in impact-parameter space. In our model we also include the most essential beyond LL corrections to BFKL. In a MC simulation these effects are resummed to all orders (see the discussion in ref. [11]). The transverse coordinate space is particularly suitable for treating unitarity constraints, multiple collisions, and saturation effects. Mueller's model includes multiple subcollisions in the frame used for the analysis, with similarities to saturation effects in the Color Glass Condensate [12-16] or the BK equation [17, 18]. In our cascade model we also include saturation effects corresponding to pomeron loops within the evolution, in a way which is almost independent of the Lorentz frame used. Also effects of confinement are included and important for the result.

In the dipole picture the colour charge in a gluon is screened by a corresponding anticharge in a neighbouring gluon. Gluon radiation implies that one dipole is split in two dipoles, and the result of the cascade is a chain of colour connected gluons. When two cascades collide, the can interact via gluon exchange. This implies an exchange of colour, producing two colour chains forming a BFKL ladder between projectile and target. Multiple interaction and saturation represent multiple pomeron exchange and pomeron loops. Note here that what is multiple interaction in one Lorentz frame is related to saturation within the cascade in another frame.

The BFKL formalism is directly applicable to inclusive observables, and the cited articles include results for total, (quasi)elastic, and diffractive cross sections in $p p$ scattering and in DIS, in very good agreement with experimental data. The numerical results are obtained with a Monte Carlo (MC) event generator called DIPSY. ${ }^{1}$

In the present paper we want to generalize the model to describe exclusive final states. Our aim is here not to generate the most precise predictions, and compete with traditional generators like PythiA 8 and HeRwig ++ . Instead we want to test our understanding of small $x$ evolution and saturation effects. In the traditional programs the evolution to small $x$ is determined by structure functions, for which the behaviour at a cutoff scale $Q_{0}^{2}$ is tuned to experimental data, and saturation effects are tuned by the value of a cutoff $p_{\perp 0}$

\footnotetext{
${ }^{1}$ Acronym for Dipoles in Impact-Parameter Space and rapidity (Y).
} 
for hard subcollisions, determined individually for every energy. In our model these effects are instead generated dynamically, based on a perturbative BFKL pomeron and screening effects from a dipole picture, including a model for pomeron loops. In addition, in our approach we can also study nontrivial effects of fluctuations and correlations, which are neglected in most analyses. In the present paper we study $p p$ collisions, but the model can also be applied to interactions with nuclei, where it gives a unique possibility to study finite size effects and effects of fluctuations. We want to return to these problems in future publications.

In order to generate exclusive states it is necessary to take into account colour coherence and angular ordering as well as soft radiation. The latter includes contributions from the $z=1$ singularity in the gluon splitting function. These effects are taken into account in the CCFM formalism [19, 20], which also reproduces the BFKL result for the inclusive cross section. In ref. [21], describing the Linked Dipole Chain model, it was demonstrated that the inclusive cross section is fully determined by a subset of the gluons in the CCFM approach, denoted " $k_{\perp}$-changing" gluons. As illustrated in figure 1 , we denote the real emitted gluons in a gluon ladder $q_{\perp i}$, and the virtual links $k_{\perp i}$. Momentum conservation here implies that $\mathbf{k}_{\perp i-1}=\mathbf{k}_{\perp i}+\mathbf{q}_{\perp i}$. In a $k_{\perp}$-changing emission the difference is large between two adjacent virtual links, $k_{\perp i-1}$ and $k_{\perp i}$. This means that $q_{\perp i} \approx \max \left(k_{\perp i-1}, k_{\perp i}\right)$.

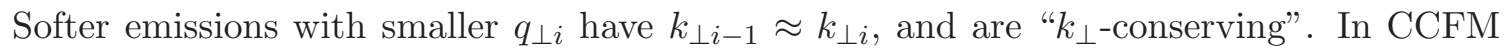
gluon emission is associated with "non-Sudakov" form factors, and in ref. [21] it was demonstrated that these gluons can be summed up in such a way, that the total cross section is fully specified by the $k_{\perp}$-changing emissions with no associated non-Sudakov form factors. The remaining $k_{\perp}$-changing gluons $q_{\perp i}^{\text {prim }}$ are called "primary gluons" in ref. [21] and "backbone gluons" in ref. [22]. They are ordered in both lightcone variables $q_{+}$and $q_{-}$, and therefore also in rapidity or angle, in accordance with QCD coherence. (In the LDC

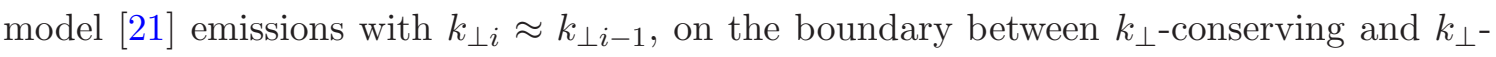
changing emissions, were treated slightly different from the BFKL prescription, which also implied a slightly different value for the exponent $\lambda$ in the power-like increase $\propto 1 / x^{\lambda}$. In the present model the LL BFKL result is exactly reproduced.)

The chains of primary gluons also determine the structure of the final state, but as discussed above, to get the full exclusive states softer emissions must be added as final state radiation. In summary the generation of exclusive final states contains the following steps:

1. The generation of two dipole cascades from the projectile and the target, in accordance with BFKL dynamics and saturation.

2. Calculating which pairs of one parton in the projectile and one parton in the target do interact. In BFKL the emission of gluons is a Poissonian type process, and the interaction probability is calculated in the eikonal approximation.

3. Extracting the primary, $k_{\perp}$-changing, gluons, and checking that they are given the correct weight. These gluons form colour connected chains between the projectile and the target, including branchings and loops. This step includes the removal of branches in the cascade which do not interact, and restoring energy-momentum conservation. 


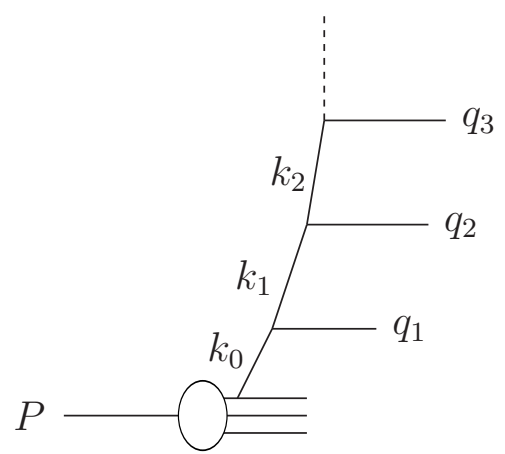

Figure 1. A chain of initial state gluon emissions. We denote the real emitted gluons in a gluon ladder $q_{\perp i}$, and the virtual links $k_{\perp i}$.

4. Adding final state radiation in relevant parts of phase space. The result consists of chains of colour connected gluons.

5. Hadronization. Here we use Lund string hadronization, where colour strings are stretched between the colour-connected gluons.

The outline of the paper is as follows: In section 2 we describe our dipole cascade model for inclusive observables. The problems encountered when generating exclusive final states are discussed in section 3. In section 4 we describe the key procedures used in the MC for solving the problems presented in section 3, while some of the more technical details are left for the appendices. Some results and predictions are given in section 5, together with a discussion of tunable parameters in the program. We conclude with a summary and outlook in section 6 .

\section{The Lund dipole cascade model for inclusive cross sections}

\subsection{Mueller's cascade model and the eikonal formalism}

Mueller's dipole cascade model [8-10] is a formulation of LL BFKL evolution in transverse coordinate space. Gluon radiation from the colour charge in a parent quark or gluon is screened by the accompanying anticharge in the colour dipole. This suppresses emissions at large transverse separation, which corresponds to the suppression of small $k_{\perp}$ in BFKL. For a dipole $(\boldsymbol{x}, \boldsymbol{y})$ the probability per unit rapidity $(Y)$ for emission of a gluon at transverse position $\boldsymbol{z}$ is given by

$$
\frac{d \mathcal{P}}{d Y}=\frac{\bar{\alpha}}{2 \pi} d^{2} \boldsymbol{z} \frac{(\boldsymbol{x}-\boldsymbol{y})^{2}}{(\boldsymbol{x}-\boldsymbol{z})^{2}(\boldsymbol{z}-\boldsymbol{y})^{2}}, \quad \text { with } \bar{\alpha}=\frac{3 \alpha_{s}}{\pi} .
$$

This emission implies that the dipole is split into two dipoles, which (in the large $N_{c}$ limit) emit new gluons independently, as illustrated in figure 2. The result reproduces the BFKL evolution, with the number of dipoles growing exponentially with $Y$. 


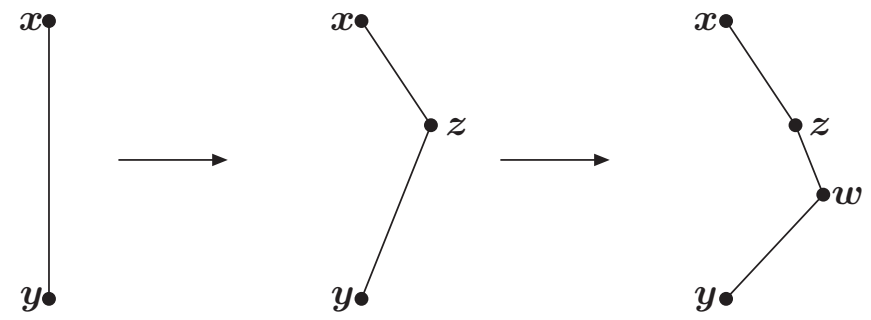

Figure 2. The evolution of the dipole cascade in transverse coordinate space. In each step, a dipole can split into two new dipoles with decay probability given by eq. (2.1).

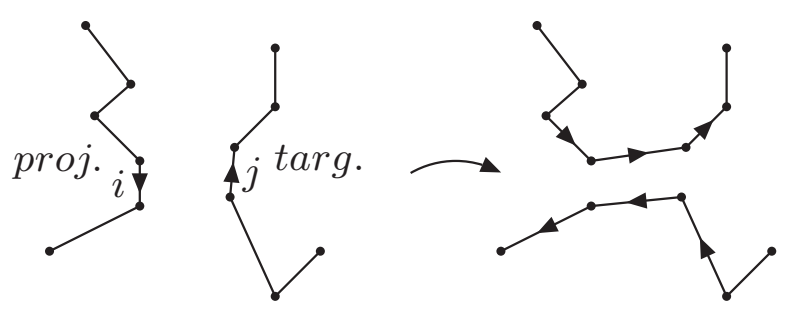

Figure 3. A dipole-dipole interaction implies exchange of colour and reconnection of the dipole chains in the colliding cascades. The arrows indicate the direction of the dipole going from colour charge to anticharge.

In a high energy collision, the dipole cascades in the projectile and the target are evolved from their rest frames to the rapidities they will have in the specific Lorentz frame chosen for the analysis. Two colliding dipoles interact via gluon exchange, which implies a colour connection between the projectile and target remnants, as indicated in figure 3 . In the Born approximation the interaction probability between one dipole with coordinates $\left(\boldsymbol{x}_{i}, \boldsymbol{y}_{i}\right)$ in the projectile, and one with coordinates $\left(\boldsymbol{x}_{j}, \boldsymbol{y}_{j}\right)$ in the target, is given by (the factor 2 in the definition of $f$ is a convention)

$$
2 f_{i j}=2 f\left(\boldsymbol{x}_{i}, \boldsymbol{y}_{i} \mid \boldsymbol{x}_{j}, \boldsymbol{y}_{j}\right)=\frac{\alpha_{s}^{2}}{4}\left[\log \left(\frac{\left(\boldsymbol{x}_{i}-\boldsymbol{y}_{j}\right)^{2}\left(\boldsymbol{y}_{i}-\boldsymbol{x}_{j}\right)^{2}}{\left(\boldsymbol{x}_{i}-\boldsymbol{x}_{j}\right)^{2}\left(\boldsymbol{y}_{i}-\boldsymbol{y}_{j}\right)^{2}}\right)\right]^{2} .
$$

At high energies the strong increase in the number of dipoles gives a large probability for multiple dipole-dipole subcollisions, and the transverse coordinate space is particularly suitable for the treatment of multiple interactions and unitarity constraints. Assuming that the subcollisions are uncorrelated, multiple collisions are taken into account in the eikonal approximation, where the probability for an inelastic interaction is given by

$$
\text { Int. prob. }=1-e^{-2 F} \text {, with } F=\sum f_{i j} \text {. }
$$

The multiple interactions produce loops of dipole chains, corresponding to the pomeron loops in the reggeon formalism. 
Assuming also that the elastic scattering amplitude, $T$, is driven by absorption into inelastic states, we find via the optical theorem

$$
T=1-e^{-F},
$$

and thus the probability for an elastic interaction given by $T^{2}$. (We use a definition such that $T$ in this case is purely real.)

To get the final result for the proton-proton cross sections we have to take averages over the projectile and target cascades, and integrate over impact parameter $\boldsymbol{b}$. For the total non-diffractive cross section we get

$$
\sigma_{\text {inel }}=\int d^{2} \boldsymbol{b}\left\langle 1-e^{-2 F(\boldsymbol{b})}\right\rangle=\int d^{2} \boldsymbol{b}\left\langle 1-(1-T(\boldsymbol{b}))^{2}\right\rangle .
$$

When the projectile has an internal structure, which can be excited, the purely elastic cross section is obtained by taking the average of the amplitude $T$, before taking the square:

$$
\sigma_{\mathrm{el}}=\int d^{2} \boldsymbol{b}\langle T(\boldsymbol{b})\rangle^{2}
$$

Taking first the square gives the total diffractive scattering (see ref. [23]):

$$
\sigma_{\text {diff }}=\int d^{2} \boldsymbol{b}\left\langle T(\boldsymbol{b})^{2}\right\rangle
$$

The cross section for diffractive excitation is consequently given by the difference

$$
\sigma_{\text {diff ex }}=\sigma_{\text {diff }}-\sigma_{\mathrm{el}}=\int d^{2} \boldsymbol{b}\left(\left\langle T(\boldsymbol{b})^{2}\right\rangle-\langle T(\boldsymbol{b})\rangle^{2}\right) .
$$

Thus diffractive excitation is determined by the fluctuations in the scattering amplitude. It is also possible to calculate e.g. the cross section for single diffractive excitation of the (right-moving) projectile by taking the average over the target cascade before, but over the projectile cascade after, squaring the amplitude. We also here have to subtract the elastic scattering, and thus get

$$
\sigma_{\mathrm{SD}}=\int d^{2} \boldsymbol{b}\left(\left\langle\langle T(\boldsymbol{b})\rangle_{L}^{2}\right\rangle_{R}-\langle T(\boldsymbol{b})\rangle^{2}\right),
$$

where $\langle\cdot\rangle_{L(R)}$ refers to the average over the left (right) cascade only.

\subsection{The Lund dipole cascade model}

The Lund dipole cascade introduces a number of corrections to Mueller's original formulation, which are all beyond the leading logarithmic approximation. The corrections are described in greater detail in previous articles [3-5], and a short summary is presented here.

\subsubsection{Beyond LL BFKL evolution}

The NLL corrections to BFKL evolution have three major sources [24]: 
Non-singular terms in the splitting function: these terms suppress large $z$-values in the individual parton branchings, and correspond approximately to a veto for $\ln 1 / z<$ $11 / 12$. It prevents the child from being faster than its recoiling parent. Most of this effect is taken care of by including energy-momentum conservation in the evolution, and ordering in the lightcone momentum $p_{+}$. To first approximation this corresponds to a veto for $z>0.5$ [21]. This cut is effectively taken into account by associating a dipole with transverse size $r$ with a transverse momentum $k_{\perp}=1 / r$, and demanding conservation of $p_{+}$in every step in the evolution. In addition this gives an effective cutoff for small dipoles, which eliminates the numerical problems encountered in the MC implementation by Mueller and Salam [25].

Projectile-target symmetry: a parton chain should look the same if generated from the target end as from the projectile end. The corresponding corrections are also called energy scale terms, and is essentially equivalent to the so called consistency constraint [26]. This effect is taken into account by conservation of both positive and negative lightcone momentum components, $p_{+}$and $p_{-}$.

The running coupling: this is relatively easily included in a MC simulation process. The scale in the running coupling is chosen as the largest transverse momentum in the vertex, in accordance with the results in ref. [27].

It is well known that the NLL corrections are not sufficient to give a realistic result for small $x$ evolution. The characteristic function $\chi(\gamma)$ has spurious singularities at $\gamma=0$ and 1 , the point $\gamma=1 / 2$ does not correspond to a saddle point, and the power $\lambda_{\text {eff }}$, determining the growth for small $x$, is negative for $\alpha_{s}=0.2$. As discussed in refs. [11, 28, 29], these problems can be cured by a resummation of subleading logs to all orders, which is executed automatically in our MC simulation.

As shown in ref. [21] the projectile-target symmetry and ordering in $p_{-}$shifts the spurious singularities in $\chi(\gamma)$ to $-\omega / 2$ and $1+\omega / 2$ (when the symmetric scale $k_{1} k_{2}$ is used in the Mellin transform). Forshaw et al. have demonstrated that if this shift is taken into account, the sensitivity to the exact size of the veto in $z$ is very small [30]. Salam has made a detailed study of different resummation schemes, and finds that the result is very stable, estimating the uncertainty to about $15 \%[11,24]$.

\subsubsection{Non-linear effects and saturation}

As mentioned above, multiple interactions produce loops of dipole chains corresponding to pomeron loops. Mueller's model includes all loops cut in the particular Lorentz frame used for the analysis, but not loops contained within the evolution of the individual cascades. As for dipole scattering the probability for such loops is given by $\alpha_{s}$, and therefore formally colour suppressed compared to dipole splitting, which is proportional to $\bar{\alpha}=N_{c} \alpha_{s} / \pi$. These loops are therefore related to the probability that two dipoles have the same colour. Two dipoles with the same colour form a quadrupole field. Such a field may be better approximated by two dipoles formed by the closest colour-anticolour charges. This corresponds to a recoupling of the colour dipole chains. We call this process a dipole "swing". 
Thus double dipole scattering in one Lorentz frame corresponds to a swing in another frame. This is illustrated in figure 4, which shows the collision of two dipole cascades in two different Lorentz frames. The horizontal axis represents rapidity, and the vertical axis shows symbolically the two transverse coordinates. The left column illustrates the collision in a central Lorentz frame, where the rest frame is given by the dashed vertical line, while the right column shows the same process in an asymmetric frame. Row $(a)$ shows the starting configuration with two dipoles. In the left column row $(b)$ shows the evolved dipole cascades just before the collision, row $(c)$ the situation when dipoles $(23)$ and (46) have interacted, and row $(d)$ after a simultanous interaction between (02) and (57). In the right column row (b) shows how the left cascade has a longer development, while in this example there is no emission in the right dipole. It is now assumed that dipoles (34) and (25) have the same colour, and row $(c)$ shows the cascade after a swing between these dipoles. Finally in row $(d)$ dipoles (01) and (35) have interacted. We see that the final result is the same. A double interaction in one frame is equivalent to a swing and a single interaction in another frame. We note that in this formulation the number of dipoles is not reduced. The saturation effect is obtained because the smaller dipoles have smaller cross sections. However, when as in this case, the dipoles in the loop (247) in the right figure on row $(c)$ do not interact, the process can also be interpreted as a $2 \rightarrow 1$ process, which corresponds to the emission of gluon 2 from the right cascade as seen in the central Lorentz frame.

In the MC, dipoles with the same colour are allowed to swing with a probability proportional to $\left(r_{1}^{2} r_{2}^{2}\right) /\left(r_{3}^{2} r_{4}^{2}\right)$, where $r_{1}$ and $r_{2}$ are the sizes of the original dipoles and $r_{3}$ and $r_{4}$ are the sizes of the recoupled dipoles. Dipoles with the same colour are allowed to swing back and forth, which results in an equilibrium, where the smaller dipoles have a larger weight. The given weights favour the formation of smaller dipoles, and although this scheme does not give an exactly frame independent result, the MC simulations show that it is a fairly good approximation.

\subsubsection{Confinement effects}

Confinement effects are included via an effective gluon mass, which gives an exponential suppression for very large dipoles [5]. This prevents the proton from growing too fast in transverse size, and is also essential to satisfy Froisart's bound at high energies [31].

\subsection{Initial dipole configurations}

In DIS an initial photon is split into a $q \bar{q}$ pair, and for larger $Q^{2}$ the wavefunction for a virtual photon can be determined perturbatively. The internal structure of the proton is, however, governed by soft QCD, and is not possible to calculate perturbatively. In our model it is represented by an equilateral triangle formed by three dipoles, and with a radius of $3 \mathrm{GeV}^{-1} \approx 0.6 \mathrm{fm}$. The model should be used at low $x$, and when the system is evolved over a large rapidity range the observable results depend only weakly on the exact configuration of the initial dipoles, or whether the charges are treated as (anti)quarks or gluons. 


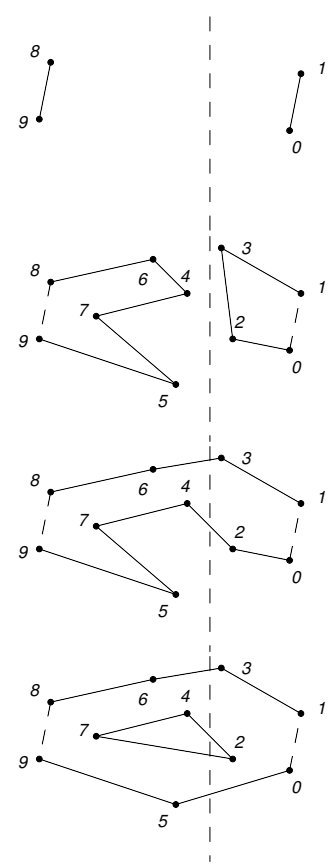

(a)

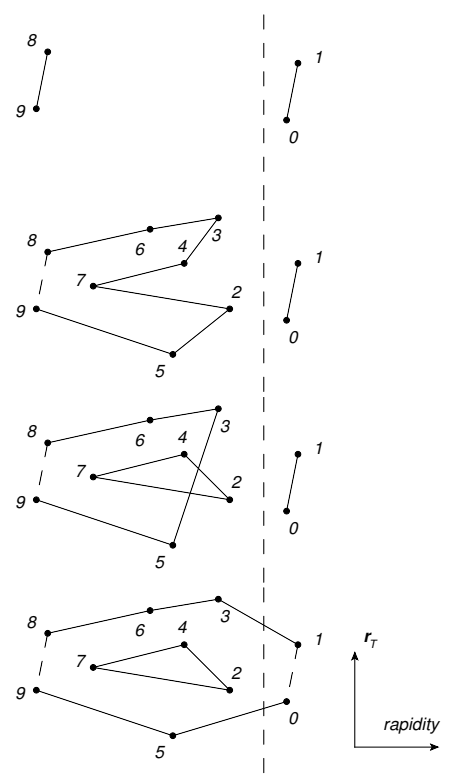

Figure 4. Creation of the same loop diagram in different interaction frames, shown as the vertical dashed lines. The horizontal axis represents rapidity, and the vertical axis the two transverse coordinates. The left side shows a central Lorentz frame where multiple interactions (dipole (23) interacting with (46) and dipole (02) interacting with (57)) forms the loop. On the right side, the same loop is formed in an asymmetric frame with a single interaction, but with a swing between (34) and (25).

\section{From inclusive to exclusive observables}

In this section we discuss the necessary steps when going from inclusive observables to exclusive final states. The technical details of the MC implementation are left to section 4 and the appendices.

\subsection{The chain of $k_{\perp}$-changing gluons}

In the introduction we discussed briefly how the structure of an exclusive final state is determined by a backbone of gluons. The first step is therefore to extract this backbone chain of $k_{\perp}$-changing or primary gluons, with their proper weights. As discussed above, the different subcollisions are assumed to be uncorrelated, and the interaction probability for two dipoles, $i$ and $j$, is given by $1-\exp \left(-2 f_{i j}\right)$, with $f_{i j}$ given by eq. (2.2). When the interacting dipoles are determined, the backbone chains can be found by tracing their parents and previous ancestors backwards in rapidity. A resulting chain is shown in figure 5a, and we here use the notation $q_{i}$ for the real emitted gluons, and $k_{i}$ for the virtual links in the evolution. The same chain is presented in figure $5 \mathrm{~b}$ in a $\left(y, \ln q_{\perp}^{2}\right)$ diagram, with $y$ being the rapidity. In this plot $\ln q_{+}$and $\ln q_{-}\left(=y \pm \ln k_{\perp}\right)$ increase towards the upper right and upper left corners. Due to the "consistency constraint" [26], which is part of the NLL corrections to BFKL, the primary gluons are ordered not only in $q_{+}$, but also in $q_{-}$. This also implies an ordering in rapidity or angle, in accordance with QCD coherence. The 
real emissions satisfy the relation $q_{i+} q_{i-}=q_{i \perp}^{2}$, and are represented by points in figure 5b. The space-like momenta for the virtual links $k_{i}$ are not constrained by such a relation, and are represented by horizontal lines indicating their transverse momentum.

The weight for a particular chain with (real) gluons $q_{i}$ is given by [21, 22] (assuming $\left.k_{\perp 0}=k_{\perp n}=0\right)$

$$
\prod_{i} \frac{\bar{\alpha}}{\pi} \frac{d^{2} q_{\perp i}}{q_{\perp i}^{2}} d y_{i} \delta\left(\Sigma \mathbf{q}_{\perp i}\right), \text { with } \bar{\alpha} \equiv \frac{N_{C} \alpha_{s}}{\pi} .
$$

We note in particular that this expression is symmetric under exchange of projectile and target. The result can also be expressed in terms of the virtual links $k_{i}$. We then have

$$
\prod_{i=1}^{n} d^{2} q_{\perp i} \delta\left(\Sigma \mathbf{q}_{\perp i}\right)=\prod_{i=1}^{n-1} d^{2} k_{\perp i},
$$

and transverse momentum conservation implies that we have two different cases:

1. Step up: $k_{\perp i} \approx q_{\perp i} \gg k_{\perp i-1}$, with weight $d^{2} q_{\perp i} / q_{\perp i}^{2} \approx d^{2} k_{\perp i} / k_{\perp i}^{2}$.

2. Step down: $k_{\perp i} \ll q_{\perp i} \approx k_{\perp i-1}$, with weight $d^{2} q_{\perp i} / q_{\perp i}^{2} \approx d^{2} k_{\perp i} / k_{\perp i-1}^{2}=d^{2} k_{\perp i} / k_{\perp i}^{2} \times$ $k_{\perp i}^{2} / k_{\perp i-1}^{2}$.

Thus steps down in $k_{\perp}$ are suppressed by a factor $k_{\perp i}^{2} / k_{\perp i-1}^{2}$. This implies that links with a local maximum $k_{\perp}$, as $k_{3}$ in figure 5 , is given the weight $d^{2} k_{\perp i} / k_{\perp i}^{4}$, and can be interpreted as a hard scattering between $k_{2}$ and $k_{4}$, producing the two high- $p_{\perp}$ gluons $q_{3}$ and $q_{4}$. For a link with $k_{\perp}$ lower than the adjacent real gluons, like $k_{4}$ in figure 5 , the transverse momentum of the real gluons will be determined by the neighbouring links $k_{3}$ and $k_{5}$. Thus in such cases the associated weight will be $d^{2} k_{\perp i}$, which is non-singular for small $k_{\perp}$. Note that no particular rest frame is specified for the collision between the two cascades. The result is the same if the "hard subcollision" is part of the projectile or target evolution, and thus independent of the Lorentz frame used for the analysis.

\subsection{Reabsorption of virtual emissions}

A dipole cascade generates a chain of dipoles linked together by gluons. A dipole-dipole interaction via gluon exchange implies exchange of colour, and the chains are recoupled as shown in figures 3 and 4 . Multiple interactions produce dipole loops, as seen in figure 4. In figure 6 we show a more complicated example, in which two cascades are evolved in rapidity up to the dashed line in the center. Here three dipole pairs interact, forming two dipole loops. In a projectile cascade with a large lightcone momentum $p_{+}$, and initially small $p_{-}$, the partons need a contribution of $p_{-}$momentum from the target, in order to come on shell. Therefore the branches denoted $B$ and $C$ in figure 6 have to be treated as virtual, and to be reabsorbed. The remaining gluons are coming on shell as real gluons, and are colour-connected along the solid lines in figure 6 . A colour loop formed by a swing, as the one denoted $A$, can come on shell also if it does not contain any interacting dipole. Such a process produces a closed colour loop not traversing the collision line at $y=0$. A more detailed example of this effect can be found in appendix C.2. The gluons in the virtual branches reabsorbed, the momenta for the real backbone gluons must be recalculated. This process is discussed in more detail in section 4 . 


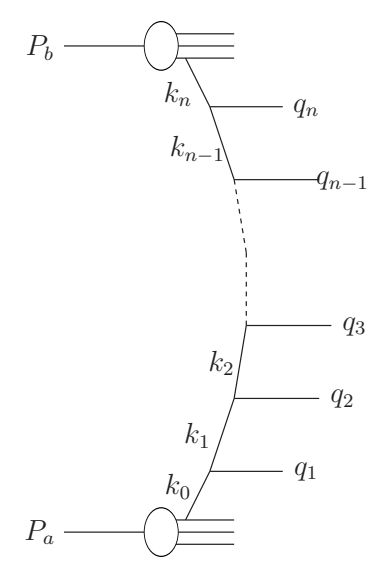

(a)

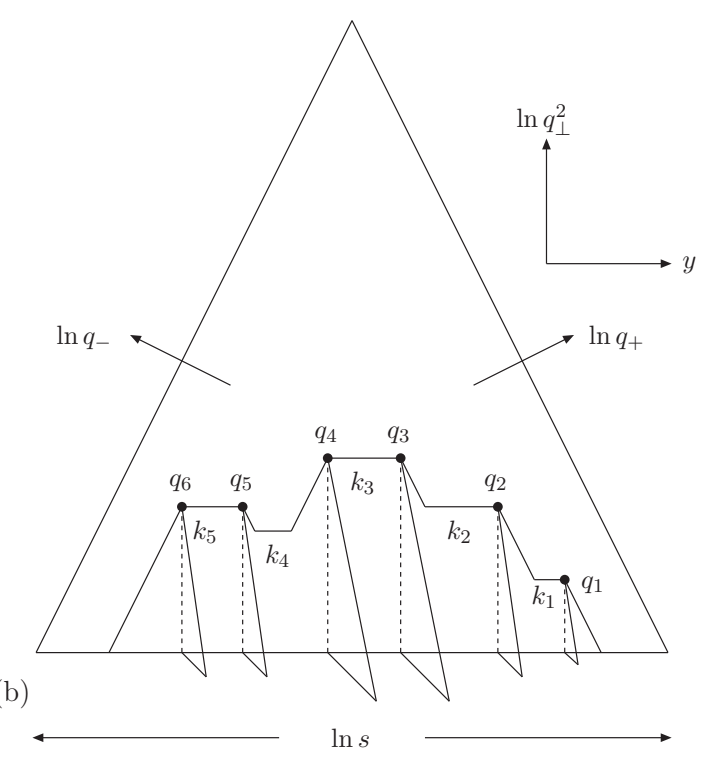

Figure 5. (a) A parton chain stretched between projectile and target. (b) A backbone of $k_{\perp}$ changing gluons in a $\left(y, \ln q_{\perp}^{2}\right)$ plane. The transverse momentum of the virtual links $k_{i}$ are represented by horizontal lines.

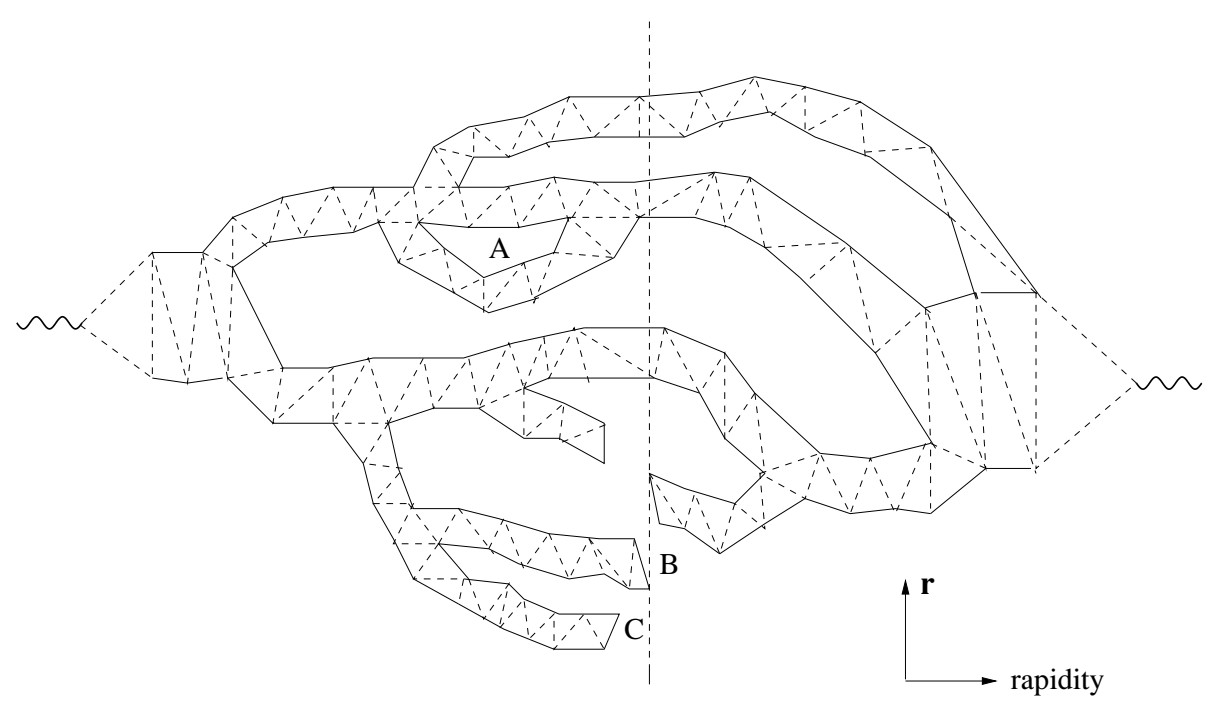

Figure 6. Collision of two dipole cascades in $\boldsymbol{r}$-rapidity space. The dashed vertical line symbolizes the Lorentz frame in which the collision is viewed. The dipole splitting vertex can result in the formation of different dipole branches, and loops are formed due to multiple sub-collisions. The loop denoted by $A$ is an effect of saturation within the cascade evolution, which can be formed via a dipole swing. Branches which do not interact, like those denoted $B$ and $C$ are to be treated as virtual, and to be absorbed. 


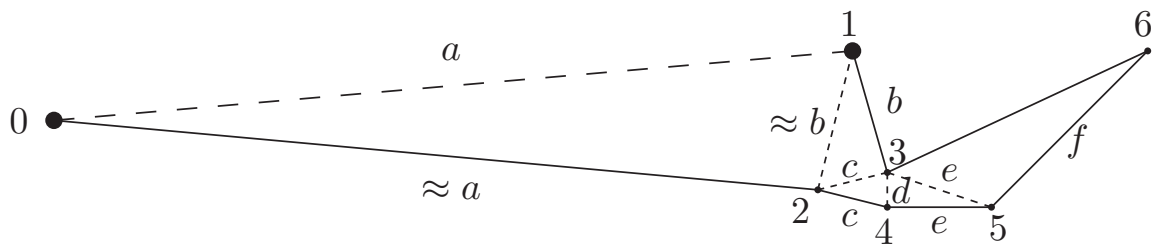

Figure 7. A dipole cascade in $\mathbf{r}_{\perp}$-space, in which a chain of smaller and smaller dipoles is followed by a set of dipoles with increasing sizes. The initial dipole between points 0 and 1 is marked by long dashes. Those dipoles which have split into two new dipoles, and thus disappeared from the chain, are marked by short dashes. The shortest dipole (34) corresponds to the maximum $k_{\perp}$, and represents a hard sub-collision.

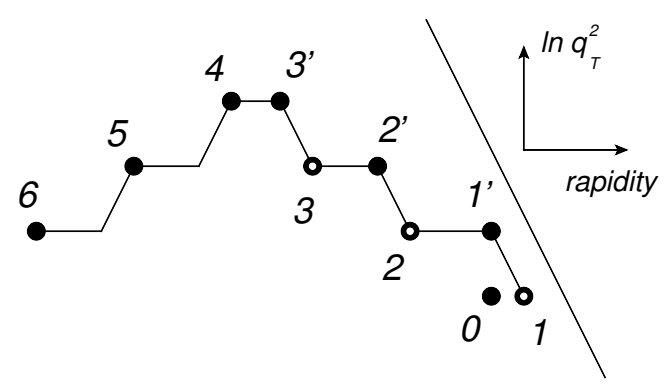

Figure 8. The same cascade as in figure 8. The $x$ direction shows rapidity, and $y$ direction shows $\ln \left(k_{\perp}^{2}\right)$. The diagonal line is the constant $p_{+}$of the incoming particle.

\subsection{Giving proper weights to the emissions}

In the cascade the gluons at the ends of a dipole are given opposite transverse momenta $k_{\perp}=1 / r$ (see further section 3.4). In eq. (2.1) an emitted dipole of length $r$ is given a weight containing the factor $d^{2} r / r^{2}$. However, if this dipole emits further dipoles, the new weight is proportional to $r^{2}$. Thus the associated weight is just $d^{2} r$ for dipoles which have split and been replaced by new dipoles. In the cascades shown in figures 7 and 9 these dipoles are marked by dashed lines. In the following they will be referred to as "inner dipoles". The remaining dipoles, formed by colour-connected gluons, are marked by heavy lines, and they all get a weight proportional to $1 / r^{2}$. They will be referred to as "outer dipoles".

Let us study the cascade shown in figure 7, obtained after the absorption of the virtual gluons as described in the previous subsection. The cascade starts from the dipole (01), followed by emission of gluons 2, 3, etc. Here the dipoles are first smaller and smaller, $a \gg b \gg c \gg d$. The corresponding $k_{\perp}$-values therefore become larger and larger in each step. After the minimum dipole, with size $d$, the subsequent emissions, 5 , and 6 , give again larger dipoles with correspondingly lower $k_{\perp}$ values. The weight containing factors $1 / r^{2}$ for all "remaining dipoles" is proportional to

$$
\frac{d^{2} \mathbf{r}_{2}}{b^{2}} \cdot \frac{d^{2} \mathbf{r}_{3}}{c^{2}} \cdot \frac{d^{2} \mathbf{r}_{4}}{d^{0}} \cdot \frac{d^{2} \mathbf{r}_{5}}{e^{2}} \cdot \frac{d^{2} \mathbf{r}_{6}}{f^{2}} \cdot \frac{1}{f^{2}}
$$




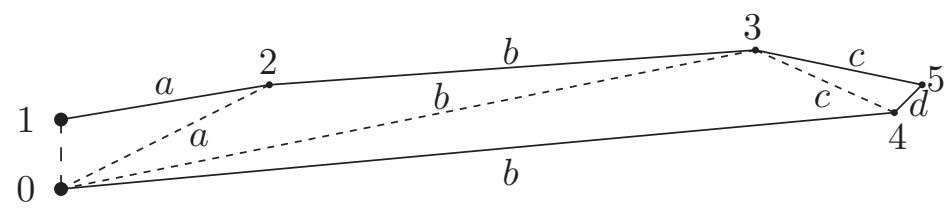

Figure 9. A cascade where the dipole sizes increase to a maximum, and then decrease. Here the size of the largest dipole, denoted $b$, corresponds to the minimum $k_{\perp}$ in the chain.

In this event gluons 3 and 4 recoil against each other with large transverse momenta $k_{\max }=1 / d$. As all factors of $d$ have canceled in eq. (3.3), this gives the proper weight $d^{2} \mathbf{r}_{4} \propto$ $d^{2} \mathbf{k}_{\max } / k_{\max }^{4}$. This reproduces exactly the proper weight for a hard gluon-gluon scattering.

The same cascade is shown in figure 8 in the $\left(y, \ln \left(k_{\perp}^{2}\right)\right)$ diagram of figure $5 \mathrm{~b}$. In this figure new emissions are first denoted by open circles, while filled circles mark their position after recoil from a later emission. Thus gluon 1 starts at the lower right corner. After the emission of gluon 2, it is recoiling to the position marked $1^{\prime}$. Similarly gluon 2 is shifted to position $2^{\prime}$, when it emits gluon 3 . When gluon 4 emits the softer gluon 5 , its recoil is small and it keeps its position in the diagram.

Figure 9 shows instead a chain with increasing dipole sizes up to a maximum value, $r_{\max }$, which thus corresponds to a minimum transverse momentum, $k_{\min }$. Here we also get the correct weight $d^{2} \mathbf{r}_{\max } / r_{\max }^{4} \propto d^{2} \mathbf{k}_{\min }$, which implies that there is no singularity for small $k_{\perp}$-values. Note that all gluons connected to the long dipoles are also connected to shorter dipoles, which determine most of their transverse momentum. Thus no gluon has $q_{\perp}$ represented by $k_{\text {min }}$.

\subsection{Going from transverse coordinate space to momentum space}

Cascade evolution. The dipole picture is formulated in transverse coordinate space, but experimental data are given in momentum space. We must therefore make a translation of our results from transverse coordinate to transverse momentum space. Note that most of the inclusive observables studied previously have been dominated by large dipoles, while the final-state observables are typically dominated by the large $q_{\perp}$, originating from the small dipoles. Thus effects such as proton size and confinement will be seen to affect observables very little, while new phenomena important for small dipoles will come into play. The one important exception to this is the deep inelastic cross section for large $Q^{2}$, which gauges small dipoles with an inclusive observable.

The weight $d^{2} r / r^{2}$ for gluon emission in the dipole picture corresponds to $d^{2} k_{\perp} / k_{\perp}^{2}$ in BFKL $[1,2]$ or DGLAP [32-34] evolution. We also found that a hard scattering, which in momentum space is proportional to $d^{2} k_{\perp} / k_{\perp}^{4}$, in the dipole picture corresponds to $d^{2} r$. These relations are consistent with the way we associate a dipole $\mathbf{r}$ with a transverse momentum $\mathbf{k}=\mathbf{r} / r^{2}$ (which also implies that $\mathbf{r}=\mathbf{k} / k^{2}$ ). Thus, although the fixed relation between $r$ and $k_{\perp}$ is inconsistent with Heisenberg's uncertainty relation, it still reproduces the correct evolution also in momentum space. This result would also be obtained by 
the relation $k_{\perp}=c / r$, for any constant $c$. (Any result for a cross section would get the same number of extra factors of $c$ in the numerator as in the denominator.) We make the choice of $c=1$ because it gives the correct $\left\langle k_{\perp}^{2}\right\rangle$ for a Gaussian distribution in $r$ : A two-dimensional distribution $\propto \exp \left(-\mathbf{r}^{2} / R^{2}\right)$ has the average $\left\langle\mathbf{r}^{2}\right\rangle=R^{2}$. The Fourier transform of the amplitude $\propto \exp \left(-\mathbf{r}^{2} / 2 R^{2}\right)$ is $\propto \exp \left(-\mathbf{k}^{2} R^{2} / 2\right)$, and thus the density is $\propto \exp \left(-\mathbf{k}^{2} R^{2}\right)$ and $\left\langle\mathbf{k}^{2}\right\rangle=1 / R^{2}$.

Dipole-dipole interaction. It is, however, not possible to make the same identification in the dipole-dipole interaction cross section from gluon exchange. The logarithmic expression in eq. (2.2) originates from a two-dimensional Fourier transform of the propagator $1 / k_{\perp}^{2}$, including interference from interactions between the equal and unequal charges in the two dipoles. If we replace $r$ by $1 / k_{\perp}$ in eq. (2.2), we would obtain a cross section which grows like $\left(d k_{\perp}^{2} / k_{\perp}^{4}\right) \cdot \ln ^{2} k_{\perp}$ for large $k_{\perp}$. Thus, taking the Fourier transform of the scattering amplitude, and then go back to momentum space via the relation $k_{\perp}=1 / r$ does not give back the original cross section in momentum space. It is then more correct to use the original scattering cross section directly in momentum space.

The amplitude for scattering of an elementary charge against a target charge at position $\mathbf{r}_{\text {target }}$ corresponds to the (two-dimensional) Fourier transform of the logarithmic Coulomb potential in two dimensions, denoted $V\left(\mathbf{r}-\mathbf{r}_{\text {target }}\right)$ :

$$
A(\mathbf{k})=\frac{1}{2 \pi} \int d^{2} r e^{i \mathbf{k r}} V\left(\mathbf{r}-\mathbf{r}_{\text {target }}\right) \propto \frac{\alpha_{s} e^{i \mathbf{k r}_{\text {target }}}}{k^{2}} .
$$

The phase is here inessential for the cross section, which is determined by $|A|^{2}$. The phase is, however, important when the target is a dipole. For scattering against a colour charge at $\mathbf{r}_{3}$ and an anticharge at $\mathbf{r}_{4}$, we get

$$
A(\mathbf{k}) \propto \frac{\alpha_{s}}{k^{2}}\left(e^{i \mathbf{k r}_{3}}-e^{i \mathbf{k r}_{4}}\right)=\frac{\alpha_{s}}{k^{2}} 2 \sin \left(\mathbf{k} \frac{\mathbf{r}_{3}-\mathbf{r}_{4}}{2}\right) e^{i \mathbf{k}\left(\mathbf{r}_{3}+\mathbf{r}_{4}\right) / 2} .
$$

For a dipole with charge and anticharge at respectively $\mathbf{r}_{1}$ and $\mathbf{r}_{2}$, scattering against a dipole with coordinates $\mathbf{r}_{3}$ and $\mathbf{r}_{4}$, we get

$$
A \propto \frac{\alpha_{s}}{k^{2}} 4 \sin \left(\boldsymbol{\rho}_{1} \cdot \boldsymbol{k}\right) \sin \left(\boldsymbol{\rho}_{2} \cdot \boldsymbol{k}\right),
$$

with $\boldsymbol{\rho}_{1}=\left(\mathbf{r}_{1}-\mathbf{r}_{2}\right) / 2$ and $\boldsymbol{\rho}_{2}=\left(\mathbf{r}_{3}-\mathbf{r}_{4}\right) / 2$. Averaging and summing over colours this gives the scattering cross section

$$
\frac{d \sigma}{d^{2} k}=8 \frac{\alpha_{s}^{2}}{k^{4}} \sin ^{2}\left(\boldsymbol{\rho}_{1} \boldsymbol{k}\right) \sin ^{2}\left(\boldsymbol{\rho}_{2} \boldsymbol{k}\right)
$$

To generate $\mathbf{k}$-values according to this distribution we simply select random uniformly distributed impact-parameters $\boldsymbol{r}_{\text {int }}$ and make the identification $\boldsymbol{k}=\boldsymbol{r}_{\text {int }} / r_{\text {int }}^{2}$. With $d^{2} r_{\text {int }}=$ $d^{2} k / k^{4}$ this corresponds to the interaction probability

$$
f_{i j}=8 \alpha_{s}^{2} \sin ^{2}\left(\frac{\boldsymbol{\rho}_{i} \cdot \boldsymbol{r}_{\mathrm{int}}}{r_{\mathrm{int}}^{2}}\right) \sin ^{2}\left(\frac{\boldsymbol{\rho}_{j} \cdot \boldsymbol{r}_{\mathrm{int}}}{r_{\mathrm{int}}^{2}}\right)
$$


Note that for small $r_{\text {int }}, f_{i j}$ goes as a constant times the rapidly oscillating sine functions, with average $(1 / 2)^{2}$. This corresponds to the expected $d^{2} k_{\perp} / k_{\perp}^{4}$ for large $k_{\perp}$. In the limit where the interaction distance is large compared to the dipole sizes, $f_{i j}$ falls off as $r_{\text {int }}^{4}$, which gives the infrared stable result $\propto d^{2} k_{\perp}$ for small $k_{\perp}$.

\subsection{Final state radiation and hadronization}

As discussed above, the backbone chain of $k_{\perp}$-changing gluons determines the inclusive inelastic cross section. To get the exclusive final states the soft $k_{\perp}$-conserving emissions have to be added as final state radiation [19, 21, 22]. As the $k_{\perp}$-conserving emissions have $q_{\perp i}<k_{\perp i} \approx k_{\perp i-1}$, they fill the area below the horizontal lines in figure 5 . Final state radiation is also emitted in the jets produced by the backbone gluons, here represented by the folds sticking out of the $\left(y, \ln q_{\perp}^{2}\right)$ plane. The separation of e.g. the colour charge in gluon $q_{3}$ and the corresponding anticharge in gluon $q_{2}$ forms a colour dipole, and gives a gluon cascade similar to the cascade in an $e^{+} e^{-}$-annihilation event. The only difference is that emissions with $q_{\perp}>k_{\perp 2}$ are not allowed.

The final-state radiation is thus added in much the same way as in the so-called LDC Monte Carlo [35, 36]. In the simulations we use the $p_{\perp}$ ordered dipole cascade model $[37,38]$ as implemented in the ARIADNE program [39]. ${ }^{2}$ In this formalism a gluon emission splits a dipole into two, both of which may continue radiating independently. Thus, also after final state radiation, the parton state consists of dipole chains formed by colour connected gluons.

In the final step the partons hadronize into final state particles. This is treated by the Lund string fragmentation model [40, 41] as implemented in the PYTHIA 8 program [42, 43]. Note that the parameters in ARIADNE have been thoroughly tuned together with the string fragmentation parameters to agree with LEP data, and we do not change any of these parameters here.

\section{Generating the exclusive final states}

The previous section outlined some of the conceptual problems encountered when describing exclusive final states in the dipole model, and how they can be solved. This section will go more into detail on how the Monte Carlo handles different situations, and how the pieces of the model are fitted together.

The first step is to select the interacting dipoles from the virtual cascade, which makes it possible to identify the on-shell and virtual gluons. The on-shell gluons will form the backbone gluons in the previous section, while the virtual gluons are removed. It will turn out that some of the backbone gluons have to be reweighted and some of the $q_{\perp}$ maxima will be removed, as will be described in section 4.3. Then a further check of ordering will be made to match phase space with final state radiation, and the colour flow between the remaining backbone gluons will be set based on the virtual cascade. These gluons will then undergo final state radiation, and hadronize to produce the final state.

\footnotetext{
${ }^{2}$ We will here use a preliminary reimplementation in THEPEG of the old ARIADNE Fortran code.
} 
Some of the more involved details, such as how the allowed phase space is chosen for emissions and interactions, and how saturation effects in the cascade and interaction complicate the procedure, will be discussed further in the appendices.

\subsection{Selecting the interactions}

As in our original Monte Carlo [3], the two incoming particles are first identified with a dipole state at their initial rapidity. These starting partons are called the valence partons, and are then evolved through rapidity to the interaction frame. Here, a relative transverse distance between the two incoming particles, the impact-parameter, is selected, and all the dipole-dipole scattering probabilities $f_{i j}$ can be calculated. Since the individual dipole interactions are assumed to be independent, eq. (3.8) can be used to find the probability for non-diffractive interaction between dipole $i$ and $j$ :

$$
1-e^{-2 f_{i j}}=1-\exp \left(-16 \alpha_{s}^{2} \sin ^{2}\left(\frac{\boldsymbol{\rho}_{i} \cdot \boldsymbol{r}_{\mathrm{int}}}{r_{\mathrm{int}}^{2}}\right) \sin ^{2}\left(\frac{\boldsymbol{\rho}_{j} \cdot \boldsymbol{r}_{\mathrm{int}}}{r_{\mathrm{int}}^{2}}\right)\right) .
$$

The interaction distance $r_{\text {int }}$ is set to the distance between two randomly selected partons, one from each dipole.

Just like in the cascade, the kinematics of the interaction is calculated. In this case, there will be two partons coming in from the left bringing with them $p_{+}$, but with a deficit in $p_{-}$, and correspondingly two partons from the right bringing $p_{-}$, but with a deficit in $p_{+}$. An interaction where the partons do not bring enough momentum to set all partons on shell is vetoed, that is $f_{i j}$ is set to 0 . The allowed phase space will be described more in detail in appendix B.

It should be noted here that although the collision of two dipoles formally is a 4-to4 reaction, with two incoming partons from each side, one of the partons in each dipole will have emitted the other parton. Thus a dipole interaction only connects the end of one backbone gluon chain, but the amplitude depends on the last two partons in the dipole picture.

\subsection{Identifying the backbone gluons}

Once the interaction dipoles are selected, one can trace the parents of the interacting partons back, and identify the backbone gluons, as illustrated in figure 6 . The rest of the partons will not get the necessary $p_{-}$from the colliding particle to come on shell, and will be reabsorbed as virtual emissions.

In a dipole picture, each emission is a coherent sum of emissions from the two partons at the end of the emitting dipole, and a unique parent cannot be determined. In the conventional parton cascade the radiation pattern is separated in two components representing independent emissions from the two different charges. The coherence effect is then approximated by angular ordering, and the recoil is taken by a single parent. For time-like cascades as in $e^{+} e^{-}$-annihilation or final state radiation this is not a dramatic effect. For the space-like cascades discussed here it is, however more important if both parents can get a recoil and be put on shell, or if the recoil effect for one of them may be so weak 

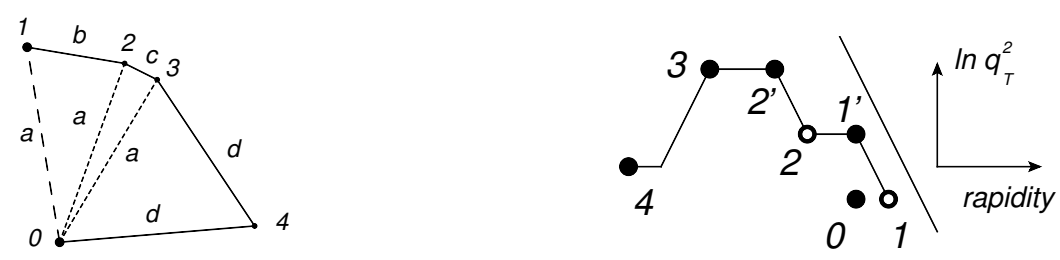

Figure 10. A cascade with a maximum in $q_{\perp}$ where the small dipole corresponding to the large $q_{\perp}$, does not split. The outer $q_{\perp}$ maximum is shown in impact-parameter space (left) and $y$ - $q_{\perp}$ space (right).

that this gluon has to be regarded as virtual and be reabsorbed. Naturally either alternative can only be approximate, and the question which of them gives the best description cannot be answered by perturbative QCD. We have therefore implemented both schemes in the event generator, and when comparing the results with experimental data we find best agreement when the parents share the recoil. For the results presented below, we have assumed that the relative shares are proportional to $1 / r_{i}^{2}$, where $r_{1}$ and $r_{2}$ are the distances to the two parents.

\subsection{Reweighting outer $q_{\perp}$ maxima}

Recalling section 3.3, two examples showed that giving the outer dipoles a weight of $d^{2} r / r^{2}$, and the inner dipoles a flat weight $d^{2} r$ reproduced the correct weights for maxima and minima in $q_{\perp}$. In these examples the minimum or maximum dipole were both inner dipoles, that is dipoles that emitted further dipoles. If an outer dipole corresponds to a local minimum as in figure 10, it is given a weight proportional to $d^{2} r / r^{2}$. As the two connected gluons both are given large transverse momenta $q_{\perp}=1 / r$, we get a distribution proportional to $d^{2} q_{\perp} / q_{\perp}^{2}$, giving a too strong tail out to large $q_{\perp}$-values.

To get the correct weights, these small outer dipoles have to be suppressed by an extra factor $r_{<}^{2} / r_{>}^{2}$, where $r_{<}$is the size of the small dipole giving the $q_{\perp}$ maximum, and $r_{>}$is the corresponding maximum in dipole size later in rapidity. We note here in particular, that this extra reweighting was not needed in the calculation of inclusive observables presented in earlier articles. These observables depend only on interacting dipoles, which are never reweighted or absorbed in this way.

In the DIPSY event generator the reweighting is implemented by finding and reabsorbing some of the outer maxima, so that the correct weight is restored. The inner and outer dipoles are only known after the backbone gluons are identified, and therefore the reweighting can be performed only after selecting the interactions and the identification of the backbone gluons. This procedure is described in more detail in appendix A.

\subsection{FSR matching and ordering}

The kinematics for the reweighted backbone gluons can be significantly different from the kinematics in the virtual cascade. To exactly match the phase space covered by ARIADne, the $q_{+}$and $q_{-}$ordering is checked again for the backbone gluons before being passed on from DIPSY. 
In ARIADNE it is then checked that each gluon in the final state cascade is un-ordered w.r.t. the backbone gluons, i.e., that the positive (and negative) light-cone momentum of an emitted gluon is less than that of (one of) the backbone gluons from which it is emitted. As the backbone gluons are required to be ordered, all of phase space is covered exactly once by the backbone gluons with FSR.

\subsection{Colour flow}

The backbone gluons are chosen and corrected looking only at the parent structure, that is, each gluon only remembers which two partons emitted it, independently of any subsequent dipole swings, as these are not changing the momenta of the backbone gluons. For final state radiation and hadronization it is, however, important to keep track of the colour flow between the backbone gluons, when the virtual emissions are reabsorbed.

This is done by going back to the colour flow of the virtual cascade, and absorb one virtual gluon at a time, until only the on-shell gluons are left. When a virtual gluon is removed, the two neighboring dipoles are combined to a single dipole. For events with no swing, this will always return the colour flow as if the virtual emissions never happened. For events with dipole swings the colour flow is more complicated, and will be discussed in appendix $\mathrm{C}$.

The proton remnant: the starting configuration of a proton is in our model represented by a triangle of three dipoles, roughly representing the positions of three valence quarks. However, three connected dipoles corresponds to the colour flow of three gluons rather than three quarks, and the extra charge may be regarded as representing the contribution from the gluons present in the proton wavefunction already at low virtuality.

This extra charge was introduced for inclusive observables, but for exclusive final states it has other effects that have to be handled. The three valence partons will in general continue down the beam-pipe, and if all three dipoles have interacted, then each of the valence partons will have two colour connections to the colliding projectile. This is an overestimate of the colour flow to the proton remnant, as the extra colour charges representing the gluonic component in general do not carry a large fraction of the proton energy. This is corrected for in DIPSY by reconnecting on average half of the colour flow to the valence partons by a dipole swing, which will move the gluonic content of the proton one "rung" down the gluon ladder.

\subsection{Higher order corrections}

The above outlines the main points of how the backbone gluons are generated in DIPSY. There are, however, further details which, despite their non-leading nature, have to be accounted for. Below follows short summaries of these corrections, while the detailed description of the algorithms implemented in DIPSY are left for the appendices.

\subsubsection{Coherence as relaxed ordering}

Previously in this section it was described how the outer $q_{\perp}$ maxima were reweighted, and absorbed with a certain probability. A $q_{\perp}$ maximum will through $q_{-}$ordering veto 
emissions at small $q_{\perp}$ close in rapidity, but if the maximum is reabsorbed, the ordered phase space will be significantly larger. This is solved by overestimating the ordered phase space in the initial generation, making it possible to emit gluons with lower $q_{\perp}$ in case the high- $q_{\perp}$ gluon is absorbed. This extra phase space can be regarded as an effect of coherence, in the sense that several close-by partons can coherently emit a gluon with a longer wavelength, and the gluon can use the combined energy in all the close-by partons. This process is described in detail in appendix B, where it is also shown how the ordering and energy conservation in the interaction is implemented, based on how it is done in the cascade.

\subsubsection{Saturation effects}

Previous considerations were all based on a single chain of dipoles, but at a $7 \mathrm{TeV}$ collision there are on average more than three subcollisions, making saturation effects very important for the final state. In appendix $\mathrm{C}$ it is shown how the procedures described here has been developed to work also in events with both multiple interaction and swings. Multiple interactions give rise to splitting chains of backbone gluons while the swing can cause both splitting and merging chains, and together they are able to build any diagram of gluon chains.

In this section it is demonstrated how the reweighting of outer $q_{\perp}$ maxima give the correct weight $d^{2} q_{\perp} \alpha_{s}^{2}\left(q_{\perp}\right) / q_{\perp}^{4}$ in every situation, with the only exception being a special configuration in two merging chains, where one of the running couplings can get an incorrect scale. This is estimated to be a negligible effect.

The colour flow in a saturated cascade is more complicated as the dipole swing will reconnect the colour flow, simulating soft gluon exchanges between different parts of the gluon chains.

\section{$5 \quad$ Self-consistency and tuning}

There are several details in DIPSY that are not fixed by perturbative calculations, but nonetheless have a significant effect on the results. These details will have to be decided by other means, or tuned to experimental data. Many of them cannot be directly related to a tunable parameter, but are rather choices between different approximations or models. Other effects were tested, only to turn out to not give improved results. Thus, rather than trying to list which parameters and choices were tuned, we will in this section describe the constraints and data, which were used to fix the details of the model. After this we will compare the model to the remaining observables.

One of the most important self-consistency constraint is the frame-independence, that is the property that all observables should be the same no matter what collision frame $y_{0}$ is used. This is a symmetry that is necessarily present in an all-order calculation, but can not be expected to be exactly manifested in a fix-order treatment as the non-leading effects enter differently in the interaction and the cascade. Thus, unknown non-leading effect will, if possible, be tuned to fit a known all-order property.

This section will first describe how the frame independence of different inclusive and exclusive observables fixes many of the details mention in section 4, and then how tuning 


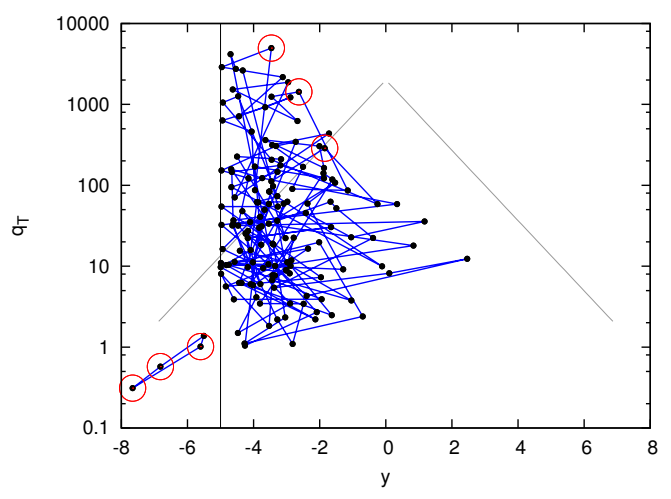

(a)

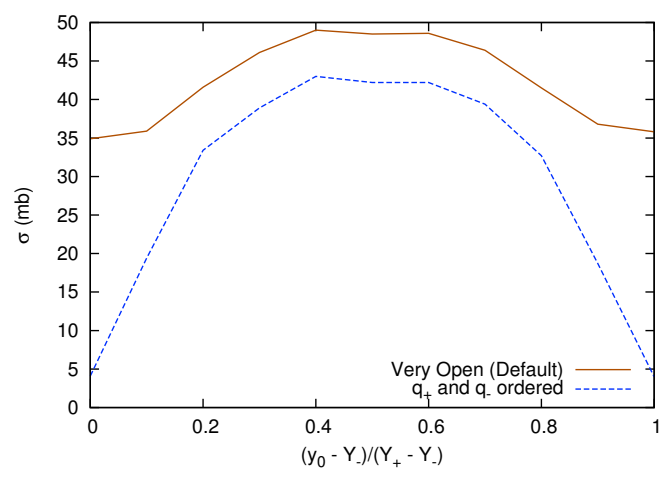

(b)

Figure 11. (a) Two colliding virtual cascades in the $y-q_{\perp}$ representation, generated by the Monte Carlo in an off-central frame. The lines shows the colour flow between the partons, the dashed vertical line is the collision frame $y_{0}$ and the circled partons are the starting valence partons. The outside triangle show the incoming $p_{+}$and $p_{-}$of the protons. (b) The frame dependence of the total $p p$ cross section at $\sqrt{s}=200 \mathrm{GeV}$. The dashed curve has full $q_{ \pm}$ordering in the interaction, the full one only requires enough energy to set the interaction on shell.

to experimental data fixes the last uncertainties. The section is ended with a comparison of DIPSY with experimental data and other event generators for a selection of observables.

\subsection{Achieving frame independence}

Here the frame dependence of some observables are studied, and many non-leading subtleties can be fixed by requiring an approximately flat frame dependence. Notice that at this stage no comparison is made to experimental data.

$\sigma_{p p}$ frame independence: we find that the restrictions in the interaction have to be very generous to maintain a constant total $p p$ cross section for $y_{0}$ close to the valence rapidities. A proton evolved over the full range to the other protons rest frame, at the Tevatron this is 15 units of rapidity, will have large transverse momenta, while the valence partons of the unevolved proton will have very small transverse momenta. Requiring full ordering in lightcone momentum in this case will disallow almost all dipole pairs, and the interaction probability will be strongly suppressed in off-central frames. This is illustrated in figure 11a with a sample virtual cascade at the Tevatron. To maintain the cross section for all frames, a very open ordering in $f_{i j}$ is needed, allowing interaction between partons even when they are not ordered from the virtual cascades. The minimum amount of ordering from appendix B.2 gives a maximum deviation of about $20 \%$ at the endpoints as can be seen in figure 11b. Anything stricter results in stronger frame dependence. The required ordering is thus set to this minimum

$$
q_{+} q_{-}>16 q_{\perp \text { int }}
$$

where $q_{+}$and $q_{-}$are the lightcone momenta the incoming partons bring, and $q_{\perp \text { int }}$ is the interaction momentum used in $f_{i j}$ in eq. (3.8). This requirement only enforces that the 


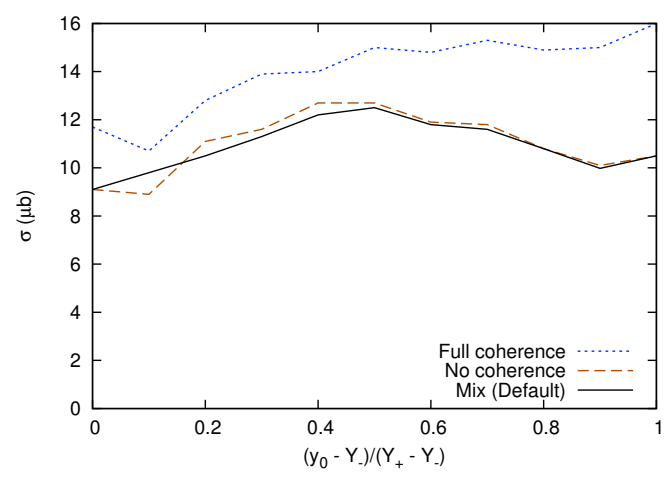

Figure 12. (a) Frame dependence of $\gamma^{*} p$ at $Q^{2}=14$ and $W=220$, showing three different orderings in the virtual cascade. For low $y_{0}$ the proton is evolved a larger part of the rapidity interval, and vice versa.

incoming particles have sufficient energy to set the interacting partons on shell, without any additional ordering. This is significantly worse than previous versions of this model, where deviations between different frames were within a few percent. This is is a sign that either the interaction ordering is too strict, blocking the interaction towards the end frames, or that the evolution does not grow fast enough, and a cascade evolved over a long rapidity range is not as strong as two cascades, each evolved only to the middle.

The first option is unlikely, as the ordering is already as generous as reasonable can be done, and in fact tests with no interaction ordering at all give no significant improvement. Likely, this problem is a result on the ordering in the virtual cascade, and how the coherence is used. During tuning it has shown that this frame dependence is quite sensitive to these choices, and further tuning could possibly solve this problem. For this tune however, the result is the one shown in figure $11 \mathrm{~b}$.

$\sigma_{\gamma^{*} \boldsymbol{p}}$ frame independences: the proton starts at a transverse momentum of about $0.3 \mathrm{GeV}$, while the two quarks from the photon start with a much higher transverse momentum. Here the key observation is that it is the $q_{+}$ordering that limits emissions of gluons with larger $q_{\perp}$, while the $q_{-}$ordering limits the emission of smaller $q_{\perp}$. Thus, with the interaction frame close to the photon, the cross section will be set by the protons ability to climb up to the higher $q_{\perp}$, dominated by the $q_{+}$ordering, and conversely, with an interaction frame close to the proton, the cross section will be determined by the $q_{-}$ ordering in the photons cascade.

This provides an opportunity to use self consistency to determine which the ordered phase space in the cascade should be. In agreement with the arguments in appendix B.1, it turns out that a fully coherent ordering will allow all emissions that may be ordered in the backbone chain. This is however done by overestimating emissions of large dipoles in the virtual cascade. This is seen in figure 12 where the coherent ordering is tilted with a larger cross section when the dipole is allowed to evolve. Here the $\gamma^{*}$ is incoming from the negative $z$-axis with a large $p_{+}$, and the proton in incoming form the positive $z$-axis with a large $p_{-}$. Ignoring coherence provides a symmetric frame dependence (although with 


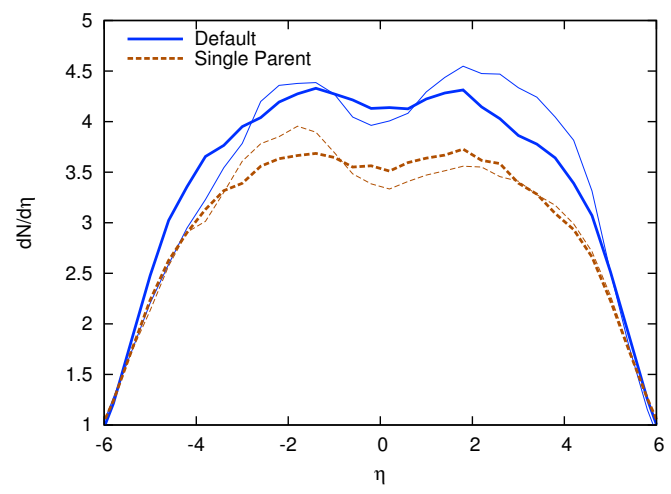

Figure 13. $d N / d \eta$ for $p p$ at $900 \mathrm{GeV}$ with default DIPSY, and DIPSY with the "single parent" setting. The thick line is collided in midframe $y_{0}=0$, the thin line in $y_{0}=-2.7$.

the same bump as in $p p$ ), but will cut away many emissions that may be ordered in the backbone chain.

A combination is used, where the $q_{\perp}$ of the effective parton is used, but the rapidity of the single parent. This maintains a symmetric frame dependence while still covering as much as possible of the ordered phase space for the backbone gluons. The $q_{-}$ordering is also very important in deciding the energy dependence for inclusive observables, such as the $p p$ total cross section. As will be seen later, it turns out that this combined ordering provides an energy dependence that fits very well with experimental data.

Frame independence of $\boldsymbol{d} \boldsymbol{N} / \boldsymbol{d} \boldsymbol{\eta}$ : for this observable to be frame independent it is needed that any given rapidity produces the same density of charged particles independently of where the interaction frame is. This means that the $q_{\perp}$ must behave the same coming from the cascade or from the interaction, and it should not depend on whether the cascade goes on for long, or if it interacts immediately. All this depends heavily on the ordered phase space in the virtual cascade and interaction, and in how the backbone gluons are handled. In practice, tuning for frame independence of $d N / d \eta$ is equivalent to balancing the $q_{\perp}$ in the interaction and in the cascade. If the interaction has too much transverse activity, then the charge particles will tend to clump up around the interaction frame when it is moved. Conversely, if the cascade provides too much $q_{\perp}$, then the side with the longer cascade will have more charged particles, and the interaction on the other side in rapidity will not be enough to balance this.

With the virtual cascade and $f_{i j}$ fixed from the inclusive observables according to the above arguments, it is only the handling of the backbone gluons left to tune. The problems connected to the reweighting of the outer $q_{\perp}$ maxima mainly entered through the ordered phase space in the virtual cascade. Once the virtual cascade and interactions are determined, the ordering of the backbone gluons is fixed by matching to FSR, so few choices are left at this point.

One choice, though, is whether both or just one parent should come on shell, as was described in section 4.2. Keeping both parents will give more activity in the cascade as more particles will be kept, and more recoils will be done. Thus, the single parent choice 

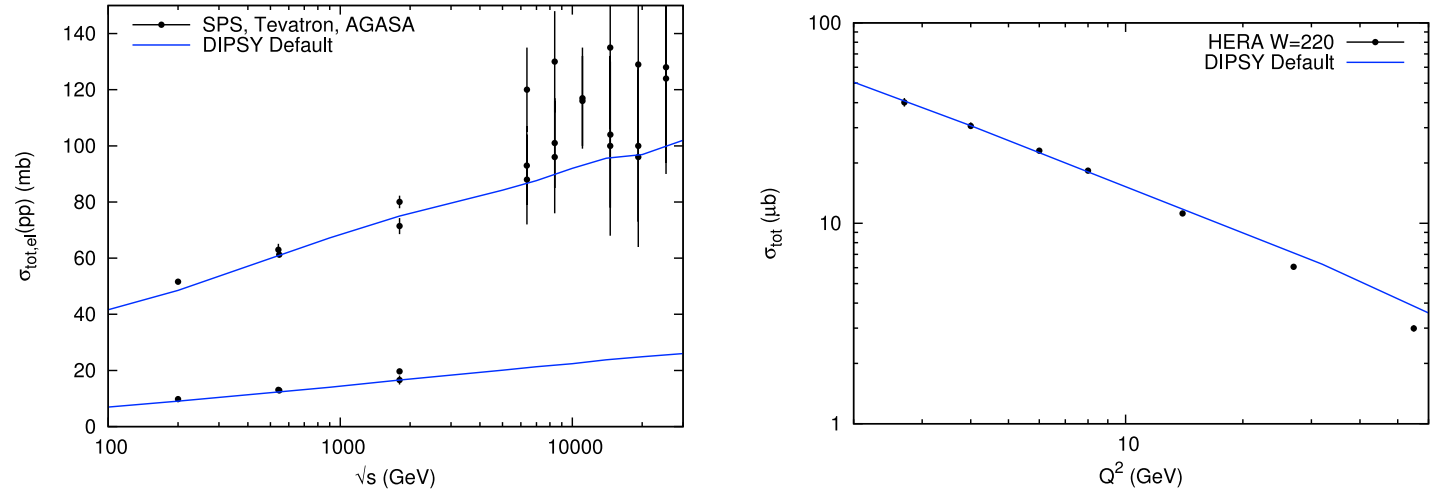

Figure 14. The inclusive data that was tuned to. (Left) The total and elastic cross section as function of $\sqrt{s}$ for $p p$. Experimental data from [44-52]. (Right) The total $\gamma^{\star} p$ cross section as function of $Q^{2}$ for $W=220 \mathrm{GeV}$. Experimental data from [53-55].

will have the multiplicity more towards the interaction side, and the double parent choice more towards the cascade side, as is seen in figure 13.

Neither of them deviate more than $10 \%$ at any pseudo-rapidity which is encouraging for the model, but neither of the options is much better than the other, so this observable cannot fix this choice. It will instead be tuned to the experimental value of this observable, which is close to line for double parent.

\subsection{Tuning to experimental data}

Inclusive $\boldsymbol{p} \boldsymbol{p}$ cross sections: in the last published tune of the Monte Carlo [6], there were 4 parameters, set to:

$$
\Lambda_{\mathrm{QCD}}=0.2 \mathrm{GeV} \quad R_{\max }=2.9 \mathrm{GeV}^{-1} \quad R_{p}=3.0 \mathrm{GeV}^{-1} \quad W_{p}=0 \mathrm{GeV}^{-1},
$$

being the QCD scale, the confinement range, the proton size, and the fluctuation in the proton size. These parameters were tuned to the total and elastic $p p$ cross section as function of $\sqrt{s}$, and the elastic differential cross section as function of $t$.

In principle the same can be done now, but one of the four parameters now hold a more important role than in previous papers. Recall from the discussion in section 3.4 that the large dipoles will dominate the inclusive $p p$ cross section, while the small dipoles will dominate the exclusive observables. One should note that $R_{\max }, R_{p}$ and $W_{p}$ mainly affect large dipoles, while $\Lambda_{\mathrm{QCD}}$ also affects small dipoles. Thus the inclusive $p p$ cross section can be tuned using $R_{\max }, R_{p}$ and $W_{p}$ without affecting the exclusive observables much. A comparison of the default tune with data can be found in figure 14 .

It should be noted that further fluctuations have been included in the proton wavefunction other than the fluctuation in size. First, the partons are allowed to fluctuate in shape, making the normally equilateral triangle of valence partons distorted. This has essentially the same effect as the fluctuation is size as a flatter triangle will have a smaller cross section. Second, the valence partons have a Gaussian smear in rapidity. While they previously were all placed at the same rapidity, that of the proton, they are now a bit spread out. This 
has a small effect also for the exclusive observables, as the peak in $d N / d \eta$ and $d p_{\perp} / d \eta$ at the valence rapidity becomes more smeared out. As in previous publications, tuning shows that all these fluctuations have to be small to maintain the ratio between the elastic and total cross section. Although the fluctuations are not needed for any observable, the fluctuations in size, angle and rapidity are set to a Gaussian distribution of widths $0.1 \mathrm{GeV}$, 0.1 and 0.1 respectively.

Inclusive $\gamma^{\star} \boldsymbol{p}$ total cross sections: as mentioned several times above, this is a key observable for tuning. Changes that affects the $\gamma^{*} p$ cross section will also affect exclusive observables such as $d N / d \eta$, and changes to the virtual cascade that affects the $d N / d \eta$ will also affect the $\gamma^{*} p$ cross section. However, changes to how the backbone gluons are handled after the virtual cascade is made, will affect only the final state, not the inclusive observables. $\sigma_{\gamma^{*} p}$ is allowing us to tune the virtual cascade for small dipoles separately, and after that is fixed, details in how the backbone gluons are treated can be tuned separately.

This observable is affected, apart from the ordering that was fixed above, by $\Lambda_{\mathrm{QCD}}$ and $c$ from section 3.4, that is the parameter that sets $q_{\perp}=c / r$. With $c=1$ as was motivated previously, the model can be made to agree with HERA data with a very reasonable ${ }^{3} \Lambda_{\mathrm{QCD}}$ of $0.23 \mathrm{GeV}$, see figure 14 .

$\boldsymbol{d} \boldsymbol{N} / \boldsymbol{d} \boldsymbol{\eta}$ : now the virtual cascade is completely fixed, and all that is left is to fix the remaining uncertainties in how the backbone gluons are selected and handled. The remaining details essentially decide how many gluons will come on shell, and how many will be reabsorbed.

The most important choice left to be fixed is the problem in section 4.2, whether one or both of a partons parents should come on shell. Not keeping both parents produce about $20 \%$ less charged particles than keeping both, and comparing experimental data, this is a bit too low. Thus we will in this tune chose to keep both parents in each emission.

This fixes all the details of the tune in this paper, and are ready to compare the tuned Monte Carlo to experimental data.

\subsection{Comparison with experiments}

We will now compare the results from our program with experimental data on exclusive final-state observables, both from the LHC and the Tevatron.

The DIPSY program is written in $\mathrm{C}++$ using ThePEG [56], which is a toolkit and framework for implementing event generation models. This framework is also used by HERWIG $++[57]$ and, more importantly here, a new version of ARIADNE [39], a pre-release of which we have been using for the results presented in the following. The Lund string fragmentation model of PythiA 8 has been interfaced to ThEPEG and is also used with DIPSY. In this way we produce exclusive hadronic final-state, which can be directly compared to data.

We will use the RIVET framework [58] (version 1.5.0) for validating event generators, which is also available from within THEPEG. We have selected some representative ob-

\footnotetext{
${ }^{3}$ For comparison, $\Lambda_{\mathrm{QCD}}=0.22 \mathrm{GeV}$ in the final state shower in ARIADNE, after a tune to LEP data.
} 
servables from underlying event, and minimum bias studies by the ATLAS experiment at center of mass energies of $900 \mathrm{GeV}$ and $7 \mathrm{TeV}[59,60]$. We also show some comparisons with results from the CDF experiment at $1.8 \mathrm{TeV}$ [61].

It should be noted that these observables are very difficult to describe also for the stateof-the-art general purpose event generators such as Pythia 8 [42] and HeRwig ++ [57], which can be plainly seen from a recent review of event generators [62] and on the web site http://mcplots.cern.ch/ where up-to-date comparisons between event generators and data are presented.

The most advanced of the general purpose event generators, PYTHIA 8, is the only one that gives a fair description of most minimum-bias and underlying-event data, and in the following we will compare our results, not only to data but also to a recent tuning of Pythia 8 (called $4 \mathrm{C}$ in [63]). In fact we will show two versions of this tuning, one which only includes non-diffractive events, and one which also includes elastic and diffractive scattering. Several experimental measurements include a correction to what is referred to non-single-diffractive events, which in principle should be directly comparable to our results. But this correction is very model-dependent (see e.g. [62] for a discussion on this) and we therefore give an indication of the size of this correction by including both nondiffractive and diffractive results from PYTHIA 8. Note that our results should be compared to the non-diffractive results from PYTHIA 8.

Also for DIPSY we show two sets of curves. As discussed above, a serious constraint for our model is that it should be independent of the Lorentz frame in which we perform the collisions. To quantify this independence we have performed simulations not only at central rapidity, where most measurements are made, but also in an asymmetric frame. ${ }^{4}$ In the central rapidity frame, the transverse momenta of final-state gluons that are relevant for the observables are predominantly given by the dipole-dipole interactions, while in the asymmetric case the transverse momenta mainly comes from recoils in the evolution.

In the following we will only show small fraction of the plots we have produced with RIVET. Further plots can be inspected on http://home.thep.lu.se/ leif/DIPSY.html.

Minimum-bias observables: in figures $15-18$ we show some standard minimum-bias observables as measure by ATLAS at $900 \mathrm{GeV}$ and $7 \mathrm{TeV}$. A general observation is that DIPSY has a slightly too weak energy dependence. Looking, e.g., in figure 15 we see that DIPSY tend to overestimate high multiplicities at low energies and underestimate them at high energies. Note, however that the cross section for e.g. multiplicities between 40 and 50 increases by a factor 40, and a large part of the increase is described by DIPSY (a factor 25). The effect is more clearly seen in the pseudo-rapidity dependence of the average multiplicity in figure 17, where again the energy dependence in DIPSY is a bit too weak.

For the transverse momentum distribution in figure 16, the energy dependence is a bit better, but DIPSY tend to overshoot both at small and large transverse momenta, while undershooting at medium values. For large transverse momenta, we believe that our description may improve if we include $2 \rightarrow 2$ matrix element corrections for local $q_{\perp}$ maxima, both in the evolution and in the interactions. For the softer part of the spectrum,

\footnotetext{
${ }^{4}$ The rapidity of the collision frame is taken to be 2.7 for $900 \mathrm{GeV}, 3.0$ for $1800 \mathrm{GeV}$, and 3.5 for $7 \mathrm{TeV}$.
} 

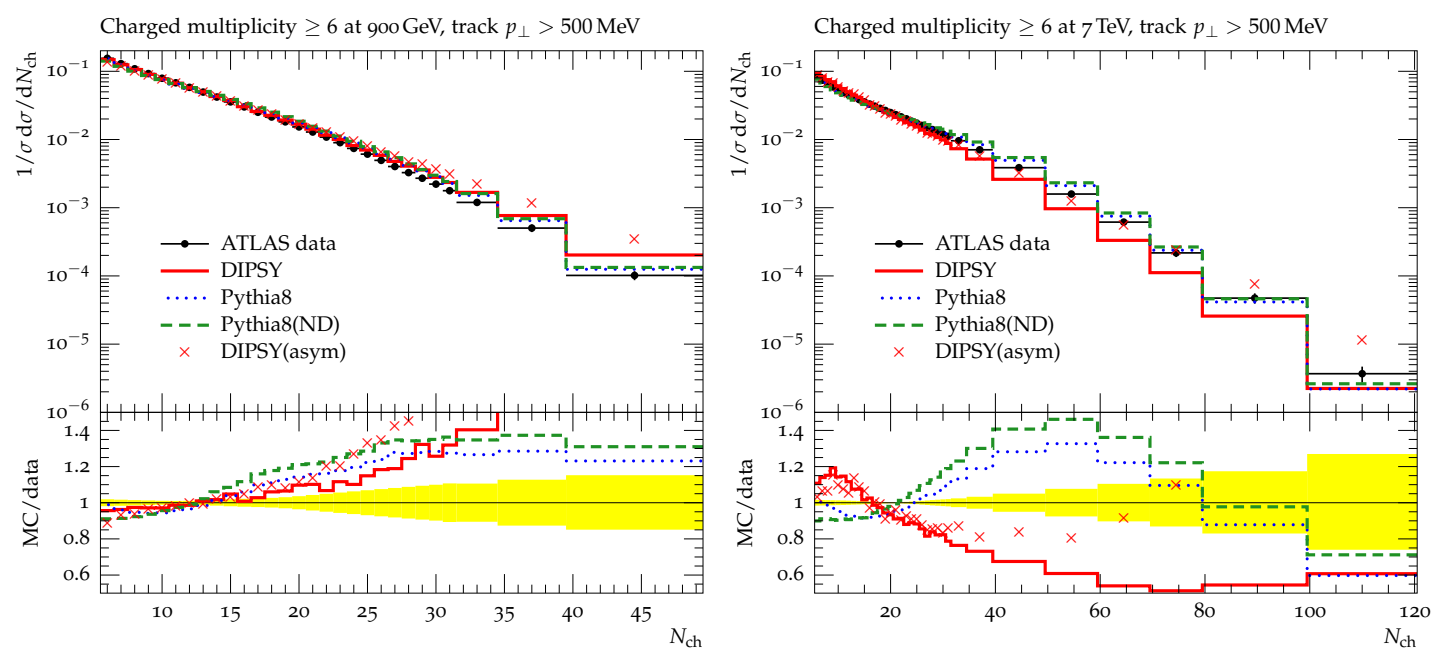

Figure 15. The charged multiplicity distribution at 0.9 (left) and $7 \mathrm{TeV}$ (right). The data points are the ones given in RIVET version 1.5.0 and are taken from [60] and include only tracks with $p_{\perp}>500 \mathrm{MeV}$ in events with more than 6 charged tracks. The full lines are the DIPSY results, the dotted lines are from PүтнIA 8 with diffractive and non-diffractive events, the dashed lines are PythiA 8 with only non-diffractive events, and the crosses are from a DIPSY simulation in an asymmetric frame.
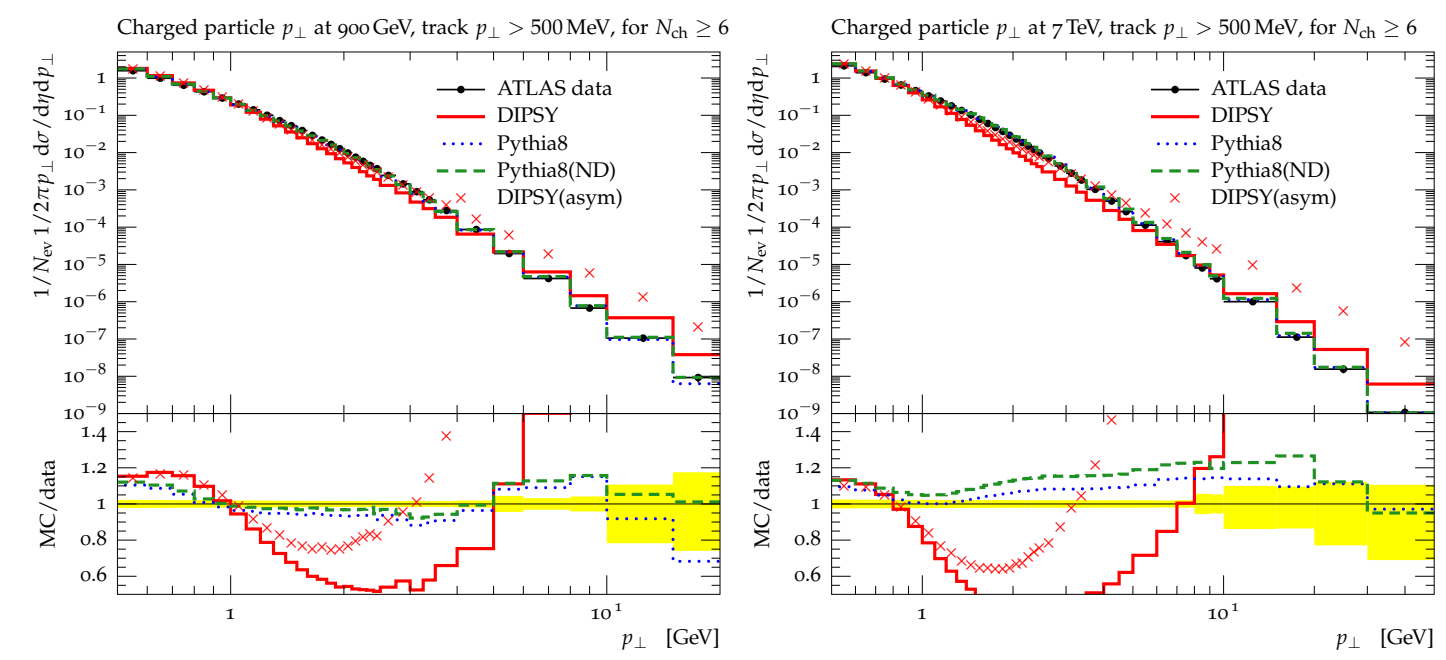

Figure 16. The transverse momentum distribution of charged particles at 0.9 (left) and $7 \mathrm{TeV}$ (right). Data points and lines as in figure 15.

we believe that the discrepancy is related to the point-like valence partons in the proton wavefunction. Even when evolved to mid-rapidity, the valence partons will still have a large $q_{+}$and will allow emissions at large $q_{\perp}$. Even though these emissions will be in midrapidity, the balancing recoil will go with the valence partons down the beam-pipe, making the spectrum in the main ATLAS detectors too soft.

In the transverse momentum distribution we also see that DIPSY in the asymmetric frame gives a much harder spectrum, indicating that the transverse momentum generated 

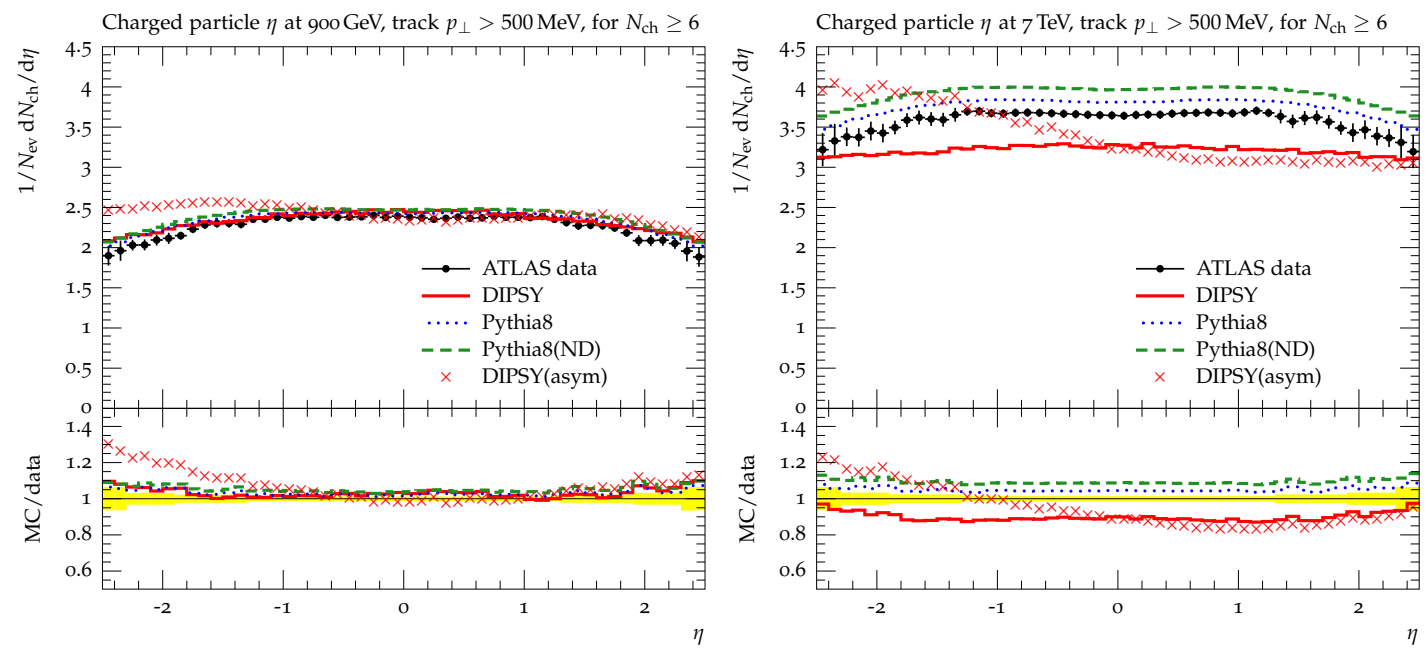

Figure 17. The pseudo-rapidity distribution of charged particles at 0.9 (left) and $7 \mathrm{TeV}$ (right). Data points and lines as in figure 15.
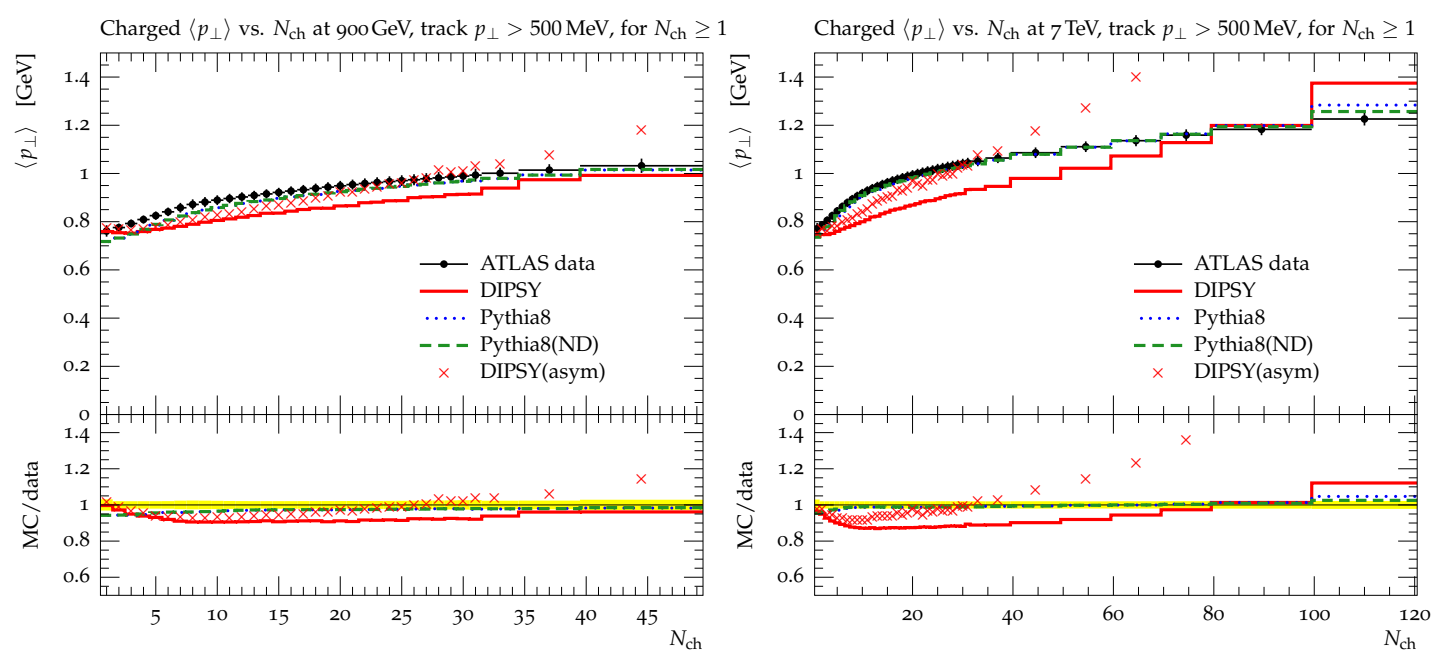

Figure 18. The average transverse momentum as a function of charged particle multiplicity at 0.9 (left) and $7 \mathrm{TeV}$ (right). Data points and lines as in figure 15 .

in the virtual cascade is harder than the what is generated in the dipole-dipole interactions. The frame dependence is also clearly seen in the pseudo-rapidity distribution in figure 17, where we see an increased activity for large rapidities in the asymmetric case, where the particles mainly originate from the evolution rather than the interactions.

In figure 18 we see that DIPSY underestimate the average transverse momentum at small and medium multiplicities, which is related to the underestimate of the number of particles with medium transverse momentum.

Underlying-event observables: in figures 19-21 we show observables related to the underlying event, many of which were introduced by Field [64]. The emphasis is to look at particle flow and transverse momenta away from jets and/or high transverse momentum 

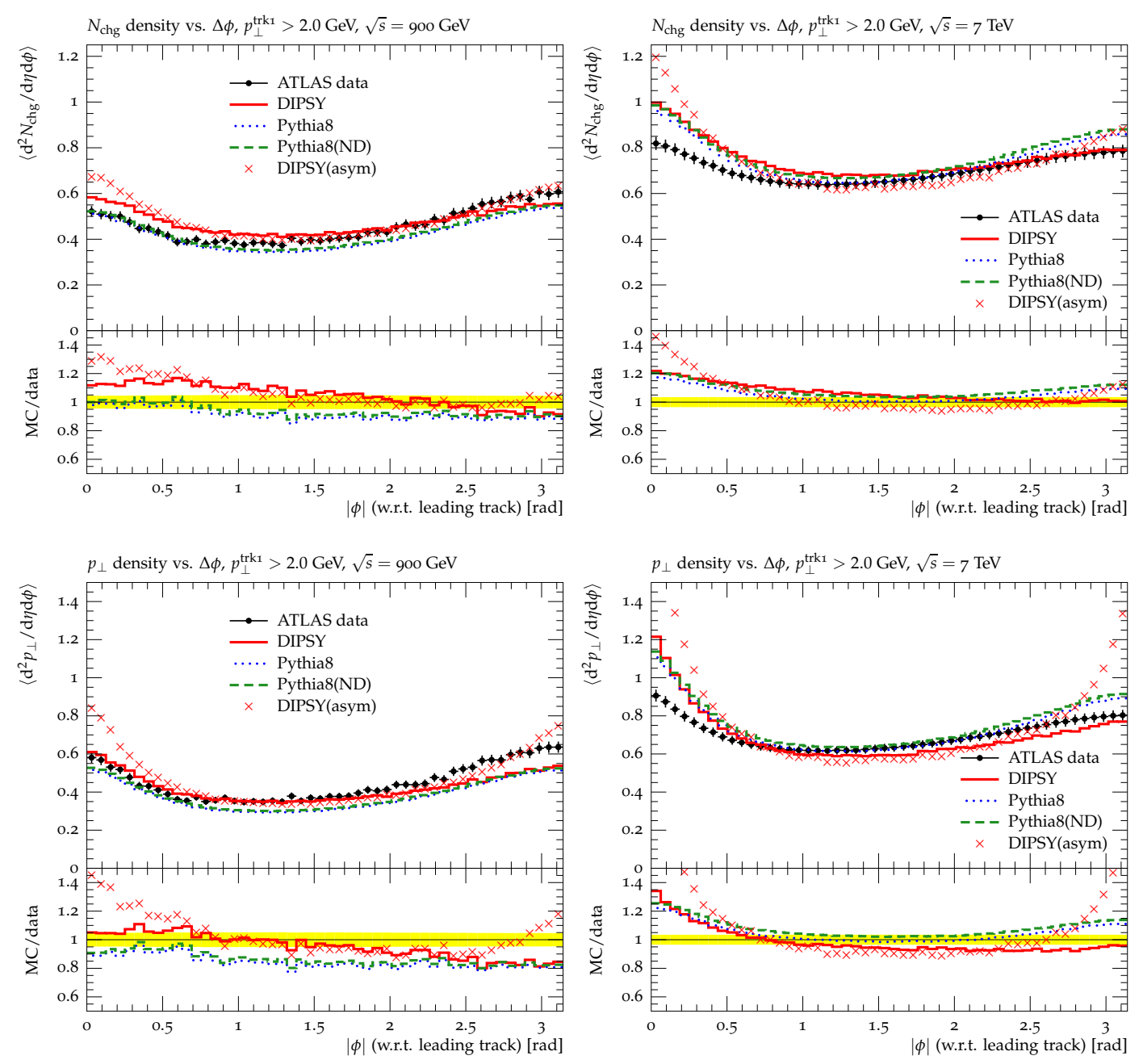

Figure 19. The multiplicity (top) and scalar sum of transverse momenta (bottom) of charge particles as a function of azimuth angle w.r.t. a leading charged particle of at least $2 \mathrm{GeV}$ transverse momenta at $0.9 \mathrm{TeV}$ (left), and $7 \mathrm{TeV}$ (right). The data points are the ones given in RIVET version 1.5.0 and are taken from [59]. The generated data as in figure 15.

charged particles to as much as possible isolate effects of the underlying events.

In figure 19 we show the multiplicity and summed transverse momentum as a function of the azimuth angle from a leading charged track with transverse momentum larger than $2 \mathrm{GeV}$. We see that all programs have difficulties reproducing the activity close to the trigger particle (the towards region) and in the opposite (away) region which should be dominated by the recoil from the leading track. In fact, all programs have similar problems, especially in the towards region, and while DIPSY in an asymmetric frame is clearly the worst, giving too much back-to-back activity, the default DIPSY actually gives a reasonable shape.

It is, however, the transverse region, defined as $60^{\circ}<\Delta \phi<120^{\circ}$, which is the most sensitive to the underlying event. In figures 20 and 21 we show the number and summed transverse momenta of charged particles in this region as a function of the transverse momentum of the leading track. We see that the PYTHIA 8 here gives a better description of data, while DIPSY as before seem to has a bit too weak energy dependence and consistently 

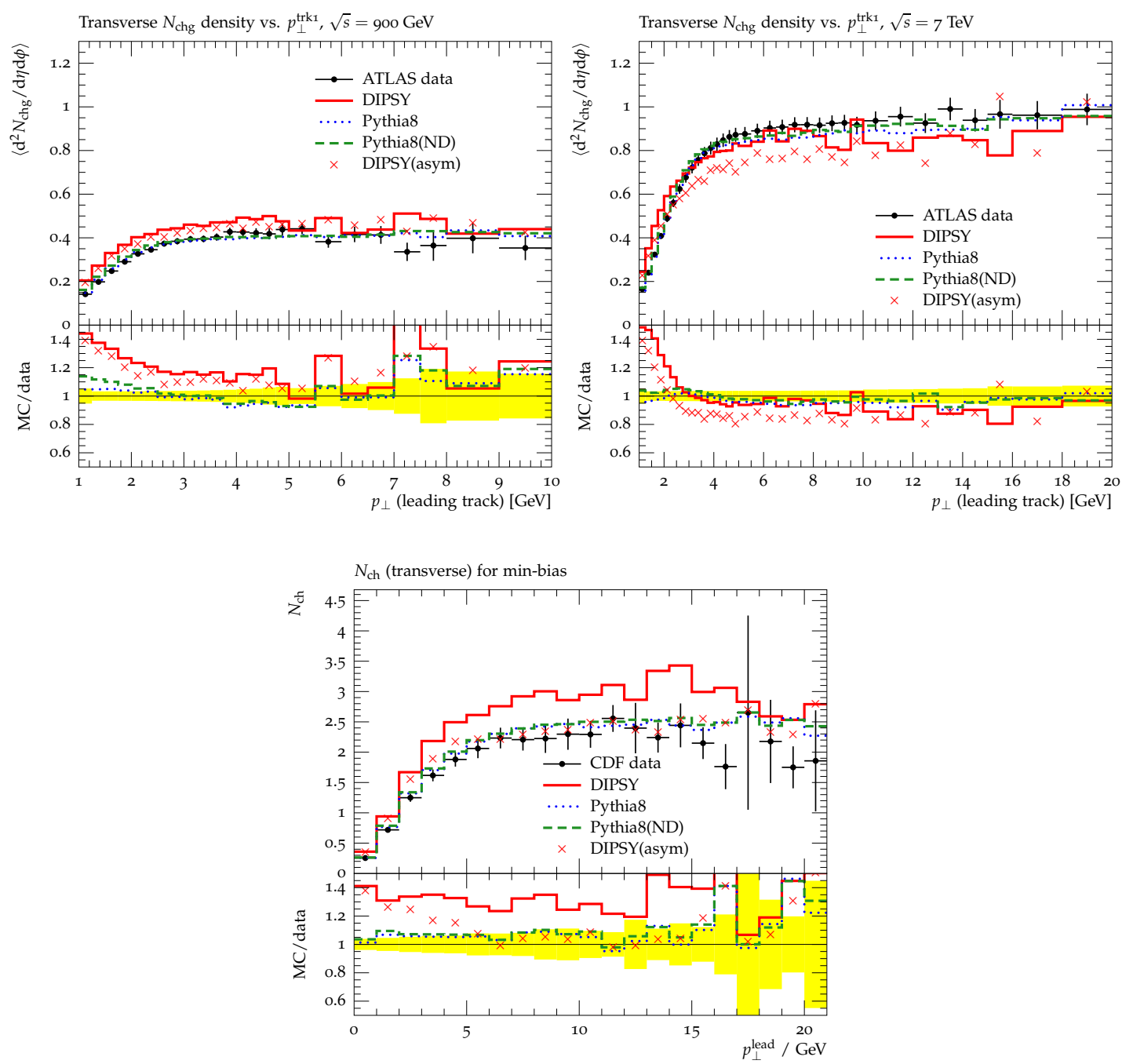

Figure 20. The multiplicity of charge particles in the transverse region, as a function of the transverse momentum of the leading charged particle at 0.9 (top left), 1.8 (bottom) and $7 \mathrm{TeV}$ (top right). Data points and lines as in figure 19. We also show data from CDF [61], but note that this data is differently normalized.

seem to overestimate the activity at small transverse momentum of the trigger particle.

\section{Conclusions}

We have here presented a completely new model for exclusive final states in non-diffractive hadronic collisions, and implemented it in a Monet Carlo program called DIPSY. ${ }^{5}$

As mentioned in the introduction our aim is not to produce the most precise model for proton collisions, but rather to study the features of small $x$ evolution and effects of

\footnotetext{
${ }^{5}$ The DIPSY program is dependent on the Ariadne program for final-state radiation. While this part of Ariadne is well tested and working, other parts of this program is still being worked on and Ariadne is therefore not officialy released. The official release of DIPSY will be done together with that of Ariadne. Meanwhile pre-releases of both DIPSY and Ariadne are available on request from the authors.
} 

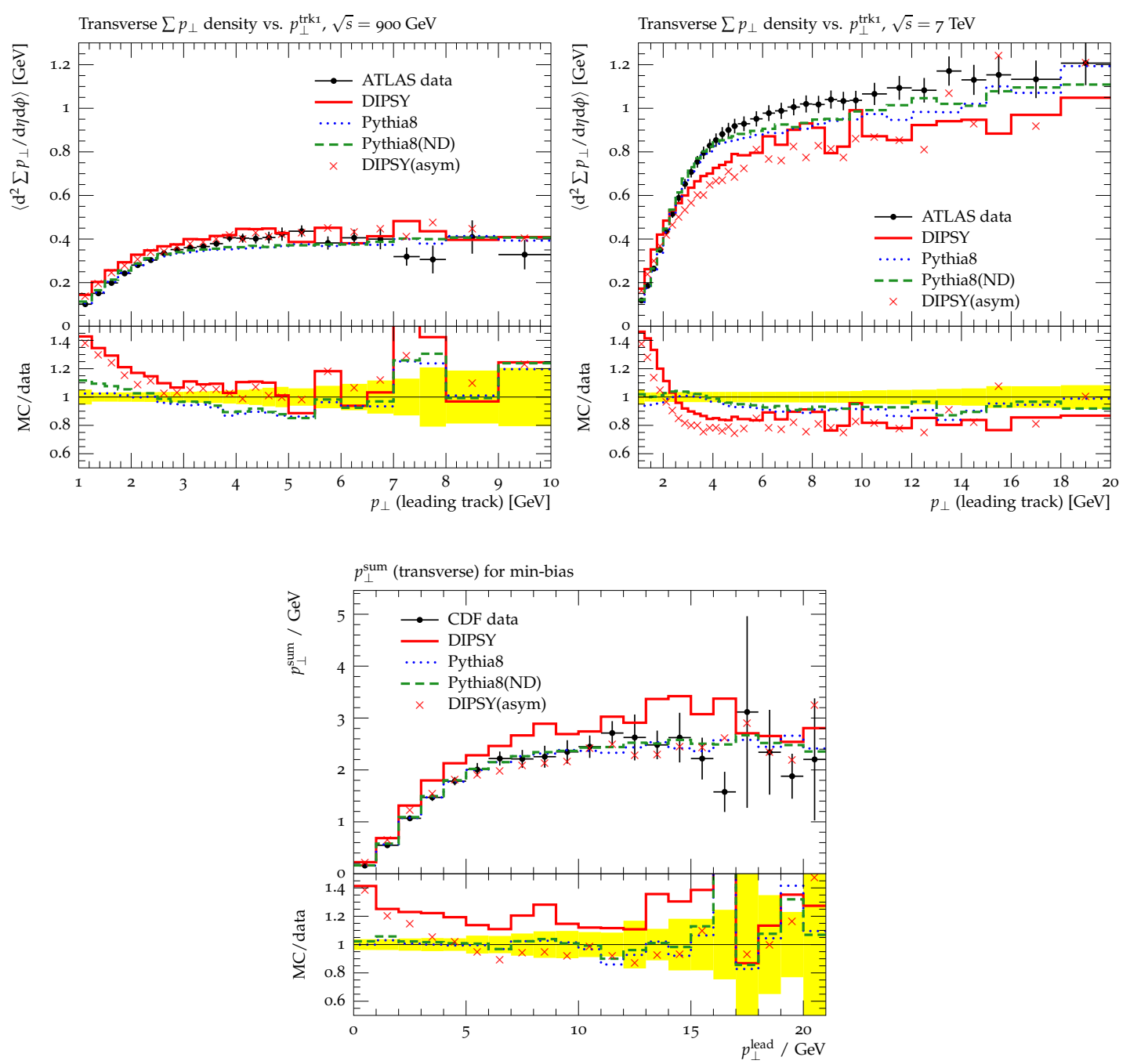

Figure 21. The scalar sum of transverse momenta of charge particles in the transverse region, as a function of the transverse momentum of the leading charged particle at 0.9 (top left), 1.8 (bottom) and $7 \mathrm{TeV}$ (top right). Data points and lines as in figure 20.

saturation. The model is based on BFKL evolution, including resummation of sub-leading logs, and saturation effects which includes pomeron loops not only due to multiple interactions but also within the cascade evolution. In earlier publications we have demonstrated that a single perturbative pomeron can describe not only total and and elastic scattering, including the scale dependence of the effective power $\lambda_{\text {eff }}\left(Q^{2}\right)$ [4], but also diffractive excitation in $p p$ collisions and DIS [5, 7]. In this paper we have extended the model to also describe exclusive final states in non-diffractive hadronic collisions.

The model is dramatically different from conventional multiple-interaction scenaria implemented in state-of-the-art general purpose event generators such as PYTHIA 8 and HERwiG++, which are based on DGLAP evolution with structure functions adjusted to experimental data. Also on the technical side, the differences are large. We use a forward evolution in impact-parameter space and rapidity, while conventional programs use back- 
ward evolution in momentum space. Our procedure has the advantage that colour screening and saturation effects are easily treated. In addition we have a detailed description of fluctuations and correlations in the proton wave function, which are typically averaged over in conventional approaches. The fluctuations are essential in diffractive excitation, and correlations are important e.g. in estimates of the effective cross section in double-parton scattering [65].

On the down-side, although the evolution of inclusive observables is rather well constrained by the resummation of sub-leading logs, the properties of exclusive states suffer from a number of non-leading effects, which influence our results. Small dipoles have little influence on inclusive cross sections, but a large effect on the final state. We have here had to make several choices, which in many cases lack guidance from perturbative QCD, and had to be determined by semi-classical and phenomenological arguments.

Throughout the the development of our model the requirement that the results should be independent of the Lorentz frame in which the interactions are performed has been a very severe constraint. In addition, our choices have been constrained by the requirement that our description of inclusive and semi-inclusive observables, such as the total and elastic cross sections remain satisfactory.

Although a big part of the framework from previous publications was reevaluated to describe exclusive observables, the part of the model that describes the inclusive observables remains basically unchanged. The reweighting of the final state gluons does not affect the interaction probability, and the ordering in the virtual cascade and interaction, which were tuned for frame-independence, are very similar to what we have presented in previous publications.

Thus, while the DIPSY program contains a fair amount of parameters and switches, maybe even as many as in the multiple-interaction model in PYTHIA 8, once the frame independence and inclusive cross sections are accounted for, there is little left that will influence the exclusive final states produced. As an example, the dependence on the collision energy of final-state observables is locked to the energy dependence of the total and elastic cross sections, and thus determined by the BFKL evolution and saturation effects. In the case of the multiple-interaction model in PythiA 8, in contrast, the energy dependence is a free parameter, although indirectly related to the increase of the total cross section with energy.

\subsection{Outlook}

Although the basic cascade evolution appears to be fairly stable, the generation of exclusive states introduces larger uncertainties, where we have to be guided by semiclassical arguments. Also, as pointed out above, the Lorentz-frame independence requirement is not quite satisfied. With this in mind, we are very pleased that although not perfect, our model gives quite reasonable results for minimum-bias and underlying-event observables at the Tevatron and the LHC. This implies that our model can be used to study if tuned parameters in conventional event generators, like e.g. the low- $p_{\perp}$ cutoff for parton subcollisions, agree with expectations for low- $x$ evolution and saturation, and if large effects of fluctuations are expected to be important. 
For future improvements we believe the most important are the matching to matrix elements for the hard subcollisions, and the inclusion of quarks. The latter is particularly important for EW processes, like $Z, W$, and Higgs production, and for forward production where our simple proton model is not expected to be sufficient.

We also plan to apply our model to collisions involving nuclei, where saturation effects are particularly important. We are here able to produce complete partonic final states in $e A, p A$, and $A A$ collision, including finite size effects and non-trivial correlations between partons in different nucleons in the nuclei. Our model does not include any hydro-dynamical evolution, but in case a quark-gluon plasma is formed, the parton state can be used as initial condition for a hydro expansion. In the absence of plasma, e.g. in $e A, p A$, or collisions between light nuclei, we are also able to generate full hadronic states. Also in collisions between heavier nuclei it can be interesting to study the collective effects by comparing the results with and without hydro.

\section{Acknowledgments}

Work supported in part by the EU Marie Curie RTN MCnet (MRTN-CT-2006-035606), and the Swedish research council (contracts 621-2008-4252 and 621-2009-4076).

L.L. gratefully acknowledges the hospitality of the CERN theory unit.

\section{A $\quad q_{\perp} \max$ reweighting in DIPSY}

As was described in section 4.3 , the maxima in in $q_{\perp}$ corresponding to an "outer" dipole in the cascade, that is a dipole that have emitted no on-shell emissions, come in with the wrong weight, and need to be reweighted.

This is in DIPSY obtained by finding and reabsorbing some of the outer maxima to restore the correct weight. The inner and outer dipoles are only known after the backbone gluons are identified, and thus the reweighting can be performed only after selecting the interactions and the identification of the backbone gluons.

To find the emissions that need to be reweighted, we note that an outer dipole corresponds to a parton that has not emitted on one side in colour flow, as parton 3 in figure 10. The maximum in $q_{\perp}$ is associated with the the backwards dipole being smaller than the forward dipole, that is, dipole $c$ being smaller than dipole $d$.

Starting from the interaction frame, the backbone gluons are checked for partons fitting these criteria, and the ones found are reweighted by an extra factor

$$
R=\frac{r_{<}^{2} \alpha_{s}\left(r_{<}\right)}{r_{>}^{2} \alpha_{s}\left(r_{>}\right)}
$$

where the running couplings have been included for better accuracy. This changes the weight of the two dipoles from $W_{\text {before }}$ to $W_{\text {after }}$, with

$$
W_{\text {before }}=d^{2} r_{<} d^{2} r_{>} \frac{\alpha_{s}\left(r_{<}\right)}{r_{<}^{2}} \frac{\alpha_{s}\left(r_{>}\right)}{r_{>}^{2}}, \quad W_{\text {after }}=d^{2} r_{<} d^{2} r_{>} \frac{\alpha_{s}^{2}\left(r_{<}\right)}{1} \frac{1}{r_{>}^{4}} .
$$



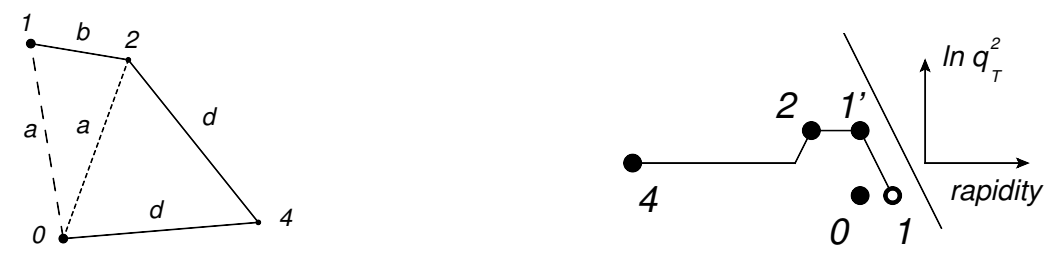

Figure 22. The backbone gluons after the outer $q_{\perp}$ maximum in figure 10 has been merged. The left figure is in impact-parameter space.

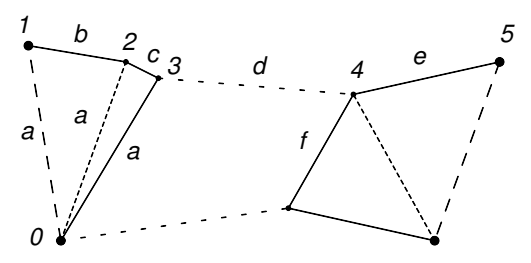

Figure 23. Partons 1 to 3 incoming from the left side collides with a cascade from the right, with partons 4 and 5. Dipole $a$ interacts with dipole $f$, which makes dipole $c$ an outer $q_{\perp}$ maximum. The figure is in impact-parameter space.

In momentum space, this corresponds to

$$
W_{\text {before }}=d^{2} q_{>} d^{2} q_{<} \frac{\alpha_{s}\left(q_{>}\right)}{q_{>}^{2}} \frac{\alpha_{s}\left(q_{<}\right)}{q_{<}^{2}}, \quad W_{\text {after }}=d^{2} q_{>} d^{2} q_{<} \frac{\alpha_{s}^{2}\left(q_{>}\right)}{q_{>}^{4}} .
$$

Notice that the small dipole size $r_{<}$is associated with the large recoil $q_{>}$, and conversely for the large dipole. The reweighting is done by reabsorbing partons of this type with a probability $1-R$, as is illustrated in figure 22. This reabsorption is done to the outer parent, so that their recoil will be canceled out, and the large $q_{\perp}$ vanish.

Some care has to be taken with the definition of an outer $q_{\perp}$ maximum close to the interacting frame, where it is not enough to look at the cascade from just one side. A dipole interacts with a probability roughly proportional to $r^{2}$, canceling out the $1 / r^{2}$ weight associated with its emission, and the colour flow will be recoupled over to the other state. Looking at figure 23 , parton 3 will not fit the above definition for outer $q_{\perp}$, as it has no emissions on either side. However, since the dipole on one side is interacting, and the dipole on the other side is not, dipole $c$ will still be a $q_{\perp}$ maximum with incorrect weight. The Monte Carlo uses a generalized algorithm that covers also these cases, and uses the dipole $d$ in figure 23 as the larger dipole to reweight and possible reabsorb parton 3.

Outer $q_{\perp}$ maxima from the interaction, for example if $d$ would be smallest dipole, does not have to be corrected as the interaction amplitude $f_{i j}$ already has the correct weight for large $q_{\perp}$.

This procedure ensures that the spectrum of large $q_{\perp}$ from the cascade and interaction is indeed going as $\frac{d^{2} q>\alpha_{s}^{2}\left(q_{>}\right)}{q_{>}^{4}}$. The proportionality constant is set from the known amplitude for a strictly increasing chain in $q_{\perp}$, and the known "penalty" for being unordered.

This extra reweighting was not needed before, as only inclusive observables were considered which are not affected by this procedure. An interacting dipole is always counted 


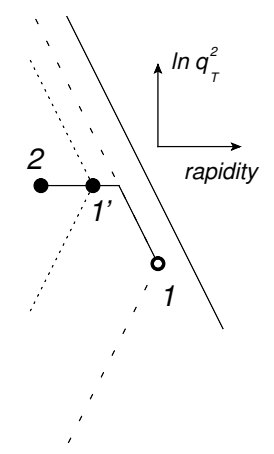

Figure 24. An emission limits the ordered phase space of the parents.

as an inner dipole in the final state, which means that no interacting dipole will be removed in this way, and the inclusive interaction probability for the two cascades is unchanged.

\section{B Absorbed partons and ordering}

In the previous sections, we describe how the backbone gluons are controlled for ordering and reweighted, and some partons are absorbed. An absorbed parton returns the $q_{+}$to its parents, and the transverse recoils are undone. This will in general move the parents back in rapidity and down in $q_{\perp}$, which opens up a larger ordered phase space for the parents as in figure 24. After parton 2 is emitted, taking part of the $q_{+}$from the parent and giving a $q_{\perp}$ recoil, 1 ' is still allowed to continue emitting. For these emissions to be ordered in $q_{+}$ and $q_{-}$, they have to be between the dotted lines in figure 24 . If parton 2 was not emitted though, the larger order phase space between the dashed lines would have been ordered with respect to 1 .

If the virtual cascade would only allow emissions ordered in $q_{+}$and $q_{-}$, every absorbed gluon would open a part of phase space for emissions from the parents that has not been considered in the virtual cascade. This is solved by partially relaxing the ordering in the virtual cascade, and thus overestimating the ordered phase space to cover the case of reabsorbed partons. Care has to be taken though, as the virtual cascade, before any reabsorbtions, will be used to calculate inclusive observables. As the backbone gluons are not yet decided when the cascade is made, it is from this approach not possible to use the correct ordered phase space already for inclusive observables, and at the same time cover all necessary phase space for all possible sets of backbone gluons.

However, by estimating what the backbone gluons may look like already in the virtual cascade one can find a sufficiently good middle way: not too strict ordering so that an important part of the possible final states get cut away, but not too open overestimate so that the inclusive cross sections inflates.

\section{B.1 Coherence}

Three observation at this point will all point towards the same solution: 


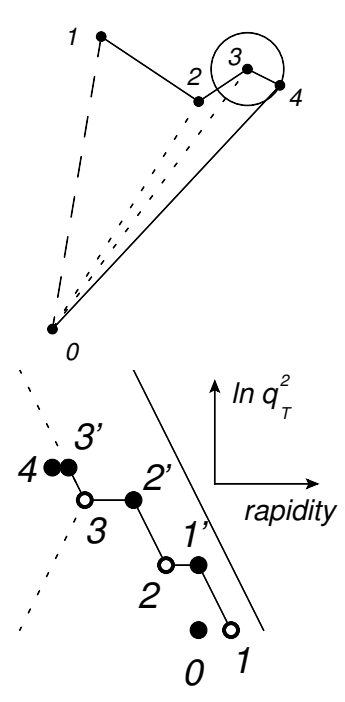

$(a)$

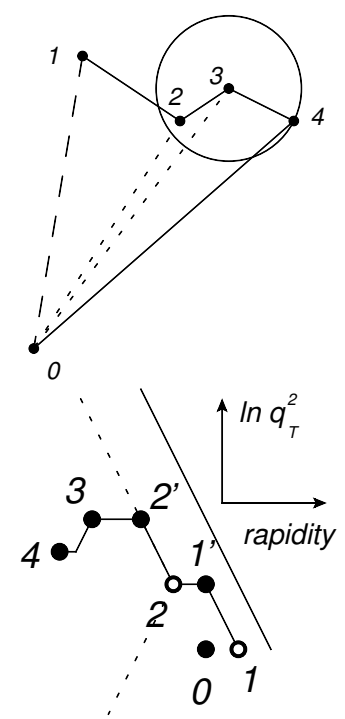

(b)

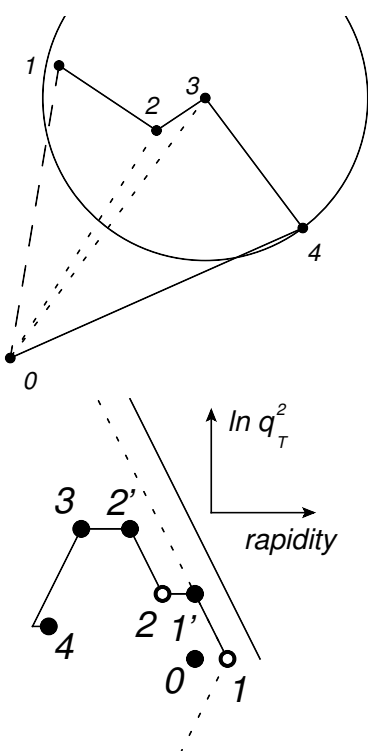

(c)

Figure 25. A parton emission (4), being emitted at different distances. The upper figures are in impact-parameter space, and the lower figures are in $y-q_{\perp}$ representation, where the dotted lines show the limits of the $q_{+}$and $q_{-}$ordering. The circles in the upper figures show the coherence range of the emission.

- Only large $q_{\perp}$ emissions, like the one in figure 24 , significantly change the parents momenta. Thus it is only absorption of a step up in $q_{\perp}$ that risks shadowing emissions from the parent.

- The phase space that get shadowed mainly concerns small $q_{\perp}$ emissions. That is, the issue is when a large $q_{\perp}$ parton is emitted and later absorbed, where the parent has not been allowed to emit with small $q_{\perp}$.

- Many of the absorbed backbone gluons are absorbed due to an outer $q_{\perp}$ maximum. This is exactly the scenario described in the previous two points, that is an absorbed step up in $q_{\perp}$ that shadows a step down in $q_{\perp}$.

In DIPSY, when a dipole is emitted in the virtual cascade, it is not only emitted by the parton at the end of the dipole, but coherently by all partons being close to the end of the dipole. This means that recoil and ordering is using an effective parton with the summed momenta of all the partons within range, where the range is set by the distance from the parent to the emitted gluon.

This procedure is shown in figure 25, where parton 4 is emitted at different distances from its parent. In $(a)$, the emission is a smaller dipole than the previous one, and the coherence range is smaller than the distance to the previous parton 2 , so the recoil and ordering will be done by parton 3 only. If the emission is at a larger distance than the previous emission, as in $(b)$, then the emission will be done by an effective parton with the merged momenta of parton 2 and 3. Notice that the recoil between 2 and 3 will cancel when 
their momenta are added, and the effective parton will have the momentum of the original parton 2, as shown in the lower figure $(b)$. Emissions at larger distance will continue to add parton to the effective parton, and can allow emissions that are not ordered with the parent itself, as in $(c)$.

An outer $q_{\perp}$ maximum is always a small dipole followed by a larger dipole, and reweighting will sometimes merge the two close by partons to one. The extra ordered phase space opened by this merging is exactly the part of phase space used by the coherent emissions at long distance, which can be seen by comparing figures 10, 22 and 25(b). This algorithm thus ensures that an absorbed outer $q_{\perp}$ maximum can not open up a part of phase space that was not considered in the virtual cascade. By solving this issue, we have been forced to introduce coherence, where an emitted gluon cannot resolve partons that are closer together than its wavelength.

While this increased ordered phase space will cover all situations in the final state, some care has to be taken. First, some of the small dipoles that emit coherently will not be absorbed, and in that case the virtual cascade has emitted large dipoles in unordered regions. These large dipoles have a significant effect on inclusive observables, and the total cross sections may get inflated.

Second, the large unordered dipoles are emitted with the motivation that the previous small dipole may get emitted. However, the situation can be that the small dipole will be absorbed because the large dipole was emitted, as the large emission makes the previous small dipole a $q_{\perp}$ maximum. Thus coherence is an estimate of how the final state will look which is to some extent self-fulfilling.

With this in mind, full coherence as described above may need to be scaled back to give a more accurate description. This was addressed in section 5.2 where frame independence was used in deciding exactly what phase space should should be considered as ordered in the virtual cascade.

\section{B.2 Ordering in the interaction}

Like with the virtual cascade it is impossible to use an ordering that allows the correct part of phase space for both inclusive and exclusive observables, but again some observations can be made.

1. The final result should not depend on whether a recoil comes from the cascade or the interaction. Thus the ordering in the interaction should allow the same backbone gluons as the ordering in the cascade.

2. It is not possible to use the exact same ordering as in the virtual cascade, as the situation is fundamentally different. At a certain rapidity in the virtual cascade, the partons have not yet gotten recoils from emissions at later rapidities. Thus, ordering in the virtual cascade is checked when only recoils with partons in one direction have been made. The same is not true in the interaction, where all recoils in both cascades are already made. 

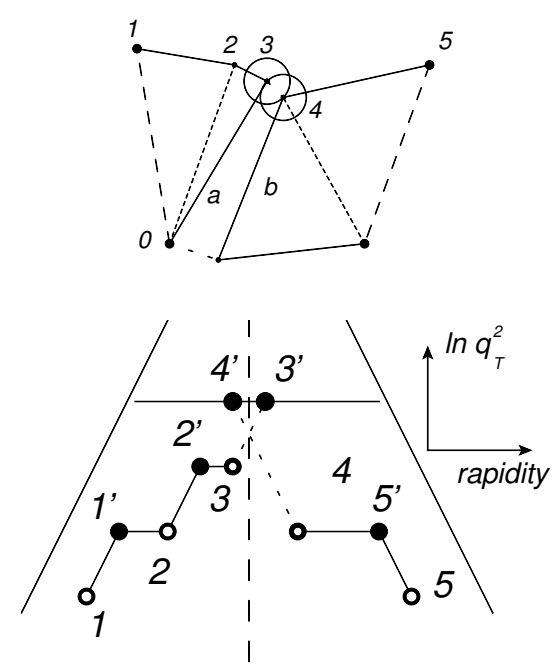

(a)
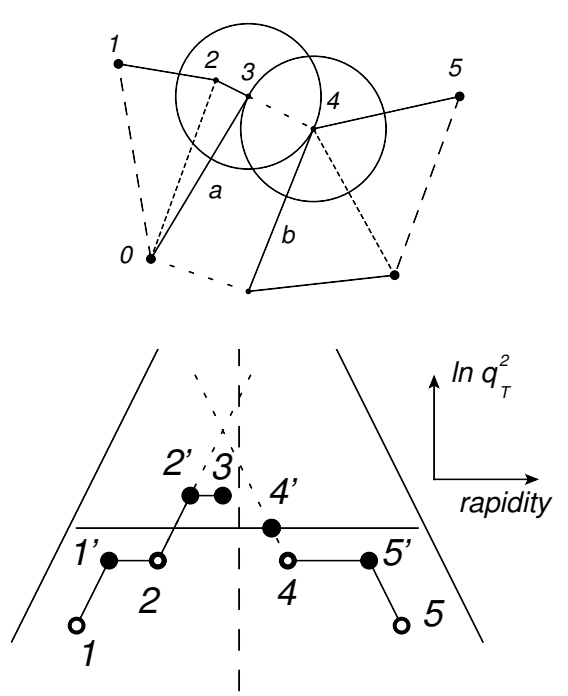

(b)

Figure 26. Ordering in an interaction for two different impact-parameters, where parton 1-3 are belonging to one cascade, and 4 and 5 to the other. The upper figures are in impact-parameter space, and the circles shows the coherence range in the interaction for parton 3 and 4 . The lower figures are in $y-q_{\perp}$ representation, where vertical dashed line is the interaction frame $y_{0}$, the horizontal full line marks the interaction $q_{\perp}$, and the diagonal dotted lines marks the $q_{-}$and $q_{+}$parton 3 and 4 respectively brings to the interaction.

3. Events where an outer $q_{\perp}$ maximum next to an interaction, as in figure 23 , is absorbed will allow more phase space for the interaction in exactly the same way as in the cascade. In the same way, coherence can be used to make sure all final states are still being considered.

4. In the virtual cascade, $q_{+}$and $q_{-}$held two fundamentally different roles where $q_{+}$was the lightcone momentum the particle came in with, and $q_{-}$measured the increasing virtuality. In the interaction the situation is completely symmetric in $q_{+}$and $q_{-}$, so the orderings must be the same.

5. In the cascade, there is no absolute limit on how small $q_{-}$can be allowed at a certain rapidity, as no momentum, $q_{+}$or $q_{-}$, will be emitted in the limit of a soft gluon. However, energy conservation sets a hard limit on how much $q_{+}$can be allowed, namely the $q_{+}$of the parent.

Putting these observations together, we see that there is a limit for how much phase space can be allowed, as there always have to be enough energy for the interaction recoil to come on shell. Notice that this corresponds to both $q_{+}$and $q_{-}$ordering, as the particles from both states must bring sufficient energy. As in the previous section, the possibility of merged partons forces coherent ordering when the interaction distance is larger than the previous dipoles in the cascade. The allowed phase space is illustrated for two different impact-parameters in figure 26.

In $(a)$, parton 3 and 4 are close to each other, corresponding to a large interaction $q_{\perp}$ that will recoil the partons past each other as can be seen in the lower figure. As 4' needs 


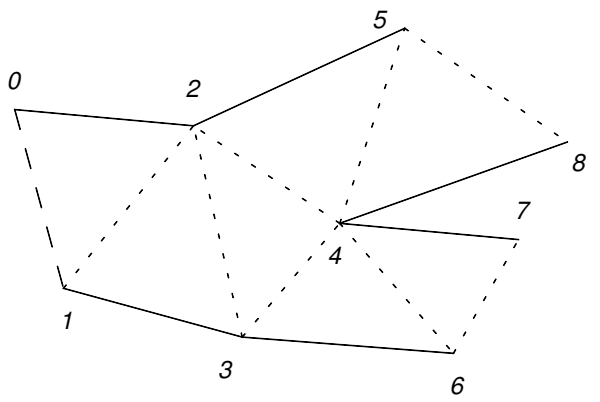

Figure 27. Two interactions makes a chain split in two. Dotted lines show parent structure, full lines show colour flow. The picture is in impact-parameter space.

more $q_{-}$than 3 can supply, and conversely parton 3' needs more $q_{+}$than 4 supplies, the interaction $q_{\perp}$ can not be set on shell, and $f_{i j}$ will be set to 0 . In this case, there is no outer $q_{\perp}$ maximum in either cascade as the maximum is in the interaction, so no parton risk being absorbed and coherence will not include any extra partons.

If the impact-parameter between the two cascades would be such as in $(b)$ where the partons 3 and 4 are farther away from each other, the interaction $q_{\perp}$ is smaller and the coherence range larger. As can be seen in the lower figure, the smaller virtuality and the coherent interaction of partons 2 and 3 means that there is enough $q_{+}$and $q_{-}$to set the interaction on shell, and $f_{i j}$ is calculated as normal.

Here it should also be taken into account that by transferring a fraction $1-z$ of the supplied $q_{+}$to the other state at fix $q_{\perp}$ will increase the virtuality by a factor $1 / z$, and conversely for the colliding parton, which gives the limit for allowed interactions:

$$
q_{+} q_{-}>16 q_{\perp \text { int }}^{2},
$$

where $q_{+}$and $q_{-}$are the supplied lightcone momenta from the interacting effective partons, and $q_{\perp \text { int }}$ is the transverse recoil from the interaction.

This is a minimum for what has to be cut away in the ordering, and from frame independence it turns out that it can not be stricter (see section 5.1), thus motivating us to set the allowed phase space in $f_{i j}$ to eq. (B.1).

\section{Saturation effects}

All motivations and examples discussed above have been based on a single backbone gluon chain, but in LHC events there will be several chains branching of and merging due to multiple interactions and the swing. This complicates the situation for most of the arguments presented previously in this section. However, the algorithms have been designed with saturation effects in mind, to give realistic results in all situations. Here follows some of the larger effects from saturation, and how the above algorithms handle them.

\section{C.1 Multiple interaction}

In the interaction, multiple scatterings are allowed, which means that there will be several chains cutting the interaction frame. These chains will merge at some point when following 


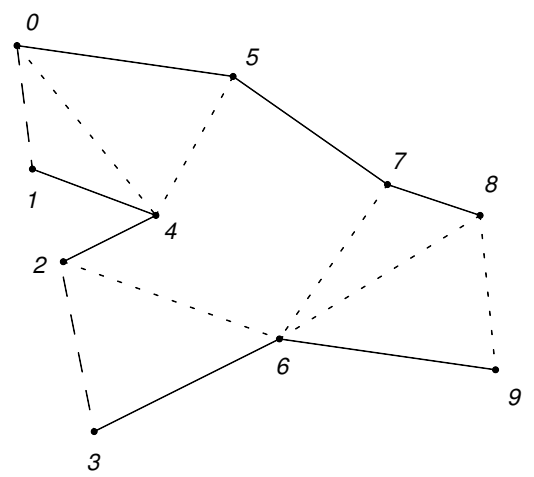

Figure 28. A swing between (45) and (26) causing two chains of backbone gluons to merge. Dotted lines show parent structure, full lines show colour flow. The picture is in impact-parameter space.

them back, if not before, they will always meet at the valence partons. This does not introduce any complications for the ordering. Each emission is controlled at the time of the emission, and if another branch has taken energy from the parents, then the ordered phase space for emission will be smaller, which is expected.

One has to be a bit careful with the reweighting however, but the algorithm described in section 4.3 is made to handle both splitting and merging chains. The dipoles created with a weight of $d^{2} \boldsymbol{r} / \boldsymbol{r}^{2}$ are still the partons that have on-shell children on one side, but not on the other, and therefore the definition of an outer $q_{\perp}$ maximum can remain.

This is illustrated in figure 27, where a backbone chain splits up in two due to multiple interaction. Dipoles (24) and (34) both get the weight $d^{2} r$ corresponding to $d^{2} q_{\perp} / q_{\perp}^{4}$, and they will get two factors of $\alpha_{s}(r)$ in case of $q_{\perp}$ maximum. Thus they do not need any extra suppression, and they do indeed not fit the definition of outer dipole, as parton 4 has children on both sides. For all other partons and dipoles, the arguments for the single chain can be applied as normal, so the reweighting algorithm works also with multiple interactions.

\section{C.2 The swing}

The dipole swing in the virtual cascade does not cause any recoils, but it does affect the parent structure of future emissions. In a non-swinged, single interaction chain, one of the two parents of each parton will also be parent to the other parent, making for the typical ladder-like structure seen in previous figures. However, with swinged emissions this is no longer true, and the two parents can have 4 different parents.

This is seen in figure 28 where a swing has recoupled dipole (45) and (26) into (24) and (56), (56) then evolves further and interacts, while (24) does not interact. Here the parents of parton 8 and 9 cover all the partons (notice that the parents of 7 are parton 5 and 6), so all partons are set on-shell. Notice in the figure that even though parton 4 is colour connected to 2 from the swing, parton 4 was not emitted by 2 (it was emitted by 0 and 1); thus no recoil has been made between the two partons, and no reweighting has to be made.

Dipole (14) has the weight $1 / r^{2}$ from the cascade and is an outer dipole as parton 4 
has no children on that side. Thus (14) will, if smaller than (45), be reweighted by

$$
\frac{r_{14}^{2} \alpha_{s}\left(r_{14}\right)}{r_{45}^{2} \alpha_{s}\left(r_{45}\right)}
$$

from eq. (A.1), and reweighting again produces the correct weights. Correspondingly for parton 2, which is in the same situation as 4 , but since 2 is a valence parton with no parents, there is no reweighting to be done. Dipole (45) is not an outer dipole because parton 7 is a child of 5 on the same side as (45), so no reweighting will be done. If (45) is a $q_{\perp}$ maximum, it will be emitted with a weight $\alpha\left(r_{45}\right) / r_{45}^{2}$, and the swing is made with a probability $\frac{r_{45}^{2} r_{26}^{2}}{r_{24}^{2} r_{56}^{2}}$, giving the weight

$$
r_{45}^{0} \alpha\left(r_{45}\right) \quad \leftrightarrow \quad \frac{\alpha\left(r_{45}\right)}{q_{45 \perp}^{4}} .
$$

Notice that while the power of $q_{\perp}$ is the correct one, a power in the running coupling is missing. This power of $\alpha_{s}$ will instead come from the smallest dipole in the emission of parton 7 from 5 and 6 . Thus, a saturated cascade can give rise to $q_{\perp}$ maxima where one of the powers in $\alpha_{s}$ is at the wrong scale.

This flaw can possibly be mended by introducing a running $\alpha_{s}$ in the swing amplitude, but in the current version of DIPSY it is not implemented. As it is a next to leading order factor in the weight occurring only in special configurations of a saturated cascade, it is estimated to be a small effect.

In conclusion, the algorithm in section 4.3 will still provide a satisfactory treatment of reweighting outer $q_{\perp}$ maxima.

\section{C.3 Colour flow in saturated cascades}

For a saturated cascade, the colour flow does not necessarily return to the original structure when the virtual emissions are removed according to 4.5. The swings are changing the colour structure, and after all the virtual emissions are reabsorbed, the colour flow does not necessarily reproduce the structure without the virtual emissions. An example of such a virtual cascade and backbone gluons is shown in figure 29.

Swings where one of the swinged dipoles go on to interact will merge two backbone chains, as was seen in section C.2, and are very important for a frame-independent description. Swings by an outer dipole, such as A, will not influence the choice of backbone gluons, but can reconnect the colour flow between the backbone gluons. A colour reconnection between backbone gluons can also happen by a swing between virtual dipoles, if the dipoles originate from different parts of the backbone chain, as is the case for swing $\mathrm{C}$. If the two swinging virtual dipoles originate from the same part on the backbone chain, as swing D, no difference will be seen in the colour flow between the backbone gluons.

In this way, not only exchange of individual soft gluons between the chains are simulated, but virtual chains of soft and hard gluons can carry colour flow between the chains, or between different parts of the same chain. The virtual cascade can thus give a very dynamic and detailed description on how the colour flow changes in saturated environments, which will be increasingly important at high energies, and can be an essential ingredient in heavy ion collisions. 

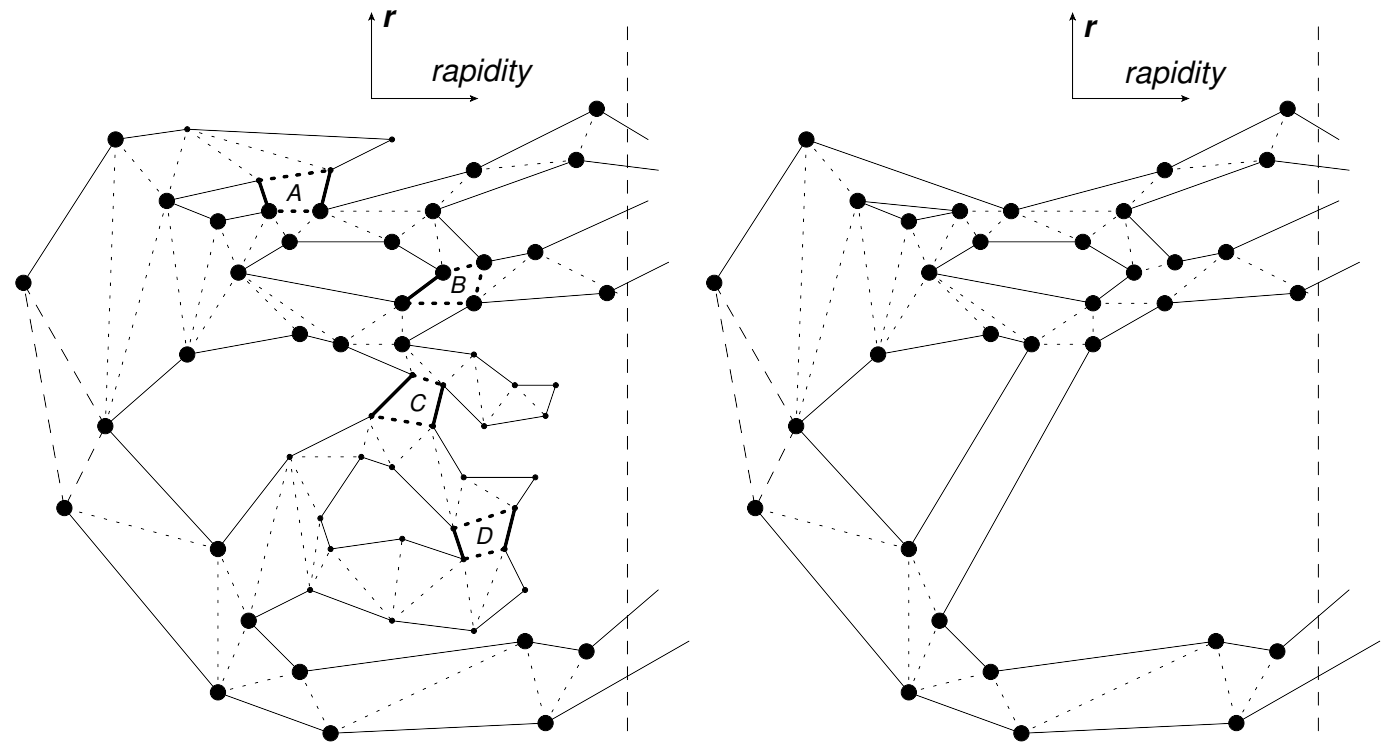

Figure 29. A cascade with swings and multiple interactions. The long dashed lines on the left side are the three valence dipoles of a proton. Full lines mark colour flow and dotted lines mark emissions. There are three interactions, which are the colour lines passing over the interaction rapidity marked by a vertical dashed line to the right. The big dots are the backbone gluons, selected through tracing each interaction back towards the valence partons, and the rest of the partons are virtual. The left figure is the virtual cascade, and four swings A to D are highlighted with thick lines. The right figure shows the backbone gluons after the virtual partons have been absorbed.

Open Access. This article is distributed under the terms of the Creative Commons Attribution Noncommercial License which permits any noncommercial use, distribution, and reproduction in any medium, provided the original author(s) and source are credited.

\section{References}

[1] E.A. Kuraev, L.N. Lipatov and V.S. Fadin, The Pomeranchuk singularity in nonabelian gauge theories, Sov. Phys. JETP 45 (1977) 199 [SPIRES].

[2] I.I. Balitsky and L.N. Lipatov, The Pomeranchuk singularity in quantum chromodynamics, Sov. J. Nucl. Phys. 28 (1978) 822 [SPIRES].

[3] E. Avsar, G. Gustafson and L. Lönnblad, Energy conservation and saturation in small-x evolution, JHEP 07 (2005) 062 [hep-ph/0503181] [SPIRES].

[4] E. Avsar, G. Gustafson and L. Lönnblad, Small-x dipole evolution beyond the large- $N_{c}$ limit, JHEP 01 (2007) 012 [hep-ph/0610157] [SPIRES].

[5] E. Avsar, G. Gustafson and L. Lönnblad, Diifractive excitation in DIS and pp collisions, JHEP 12 (2007) 012 [arXiv:0709.1368] [SPIRES].

[6] C. Flensburg, G. Gustafson and L. Lönnblad, Elastic and quasi-elastic pp and $\gamma^{*} p$ scattering in the dipole model, Eur. Phys. J. C 60 (2009) 233 [arXiv:0807.0325] [SPIRES]. 
[7] C. Flensburg and G. Gustafson, Fluctuations, saturation and diffractive excitation in high energy collisions, JHEP 10 (2010) 014 [arXiv:1004.5502] [SPIRES].

[8] A.H. Mueller, Soft gluons in the infinite momentum wave function and the BFKL Pomeron, Nucl. Phys. B 415 (1994) 373 [SPIRES].

[9] A.H. Mueller and B. Patel, Single and double BFKL Pomeron exchange and a dipole picture of high-energy hard processes, Nucl. Phys. B 425 (1994) 471 [hep-ph/9403256] [SPIRES].

[10] A.H. Mueller, Unitarity and the BFKL pomeron, Nucl. Phys. B 437 (1995) 107 [hep-ph/9408245] [SPIRES].

[11] G.P. Salam, A resummation of large sub-leading corrections at small x, JHEP 07 (1998) 019 [hep-ph/9806482] [SPIRES].

[12] E. Iancu, A. Leonidov and L. McLerran, The colour glass condensate: An introduction, hep-ph/0202270 [SPIRES].

[13] E. Iancu and R. Venugopalan, The color glass condensate and high energy scattering in QCD, hep-ph/0303204 [SPIRES].

[14] F. Gelis, E. Iancu, J. Jalilian-Marian and R. Venugopalan, The color glass condensate, Ann. Rev. Nucl. Part. Sci. 60 (2010) 463 [arXiv:1002.0333] [SPIRES].

[15] E. Iancu, A. Leonidov and L.D. McLerran, Nonlinear gluon evolution in the color glass condensate. I, Nucl. Phys. A 692 (2001) 583 [hep-ph/0011241] [SPIRES].

[16] J. Jalilian-Marian, A. Kovner, A. Leonidov and H. Weigert, The BFKL equation from the Wilson renormalization group, Nucl. Phys. B 504 (1997) 415 [hep-ph/9701284] [SPIRES].

[17] I. Balitsky, Operator expansion for diffractive high-energy scattering, hep-ph/9706411 [SPIRES].

[18] Y.V. Kovchegov, Unitarization of the BFKL Pomeron on a nucleus, Phys. Rev. D 61 (2000) 074018 [hep-ph/9905214] [SPIRES].

[19] S. Catani, F. Fiorani and G. Marchesini, Small $x$ behavior of initial state radiation in perturbative QCD, Nucl. Phys. B 336 (1990) 18 [SPIRES].

[20] M. Ciafaloni, Coherence effects in initial jets at small $Q^{2} / s$, Nucl. Phys. B 296 (1988) 49 [SPIRES].

[21] B. Andersson, G. Gustafson and J. Samuelsson, The Linked dipole chain model for DIS, Nucl. Phys. B 467 (1996) 443 [SPIRES].

[22] G.P. Salam, Soft emissions and the equivalence of BFKL and CCFM final states, JHEP 03 (1999) 009 [hep-ph/9902324] [SPIRES].

[23] M.L. Good and W.D. Walker, Diffraction disssociation of beam particles, Phys. Rev. 120 (1960) 1857 [SPIRES].

[24] G.P. Salam, An introduction to leading and next-to-leading BFKL, Acta Phys. Polon. B 30 (1999) 3679 [hep-ph/9910492] [SPIRES].

[25] A.H. Mueller and G.P. Salam, Large multiplicity fluctuations and saturation effects in onium collisions, Nucl. Phys. B 475 (1996) 293 [hep-ph/9605302] [SPIRES].

[26] J. Kwiecinski, A.D. Martin and P.J. Sutton, Constraints on gluon evolution at small $x$, Z. Phys. C 71 (1996) 585 [hep-ph/9602320] [SPIRES]. 
[27] I. Balitsky and G.A. Chirilli, NLO evolution of color dipole, Acta Phys. Polon. B 39 (2008) 2561 [SPIRES].

[28] M. Ciafaloni and D. Colferai, The BFKL equation at next-to-leading level and beyond, Phys. Lett. B 452 (1999) 372 [hep-ph/9812366] [SPIRES].

[29] M. Ciafaloni, D. Colferai and G.P. Salam, A collinear model for small-x physics, JHEP 10 (1999) 017 [hep-ph/9907409] [SPIRES].

[30] J.R. Forshaw, D.A. Ross and A. Sabio Vera, Rapidity veto effects in the NLO BFKL equation, Phys. Lett. B 455 (1999) 273 [hep-ph/9903390] [SPIRES].

[31] E. Avsar, On the high energy behaviour of the total cross section in the QCD dipole model, JHEP 04 (2008) 033 [arXiv:0803.0446] [SPIRES].

[32] V.N. Gribov and L.N. Lipatov, Deep inelastic ep scattering in perturbation theory, Sov. J. Nucl. Phys. 15 (1972) 438 [SPIRES].

[33] Y.L. Dokshitzer, Calculation of the structure functions for deep inelastic scattering and $e^{+} e^{-}$ annihilation by perturbation theory in quantum chromodynamics, Sov. Phys. JETP 46 (1977) 641 [SPIRES].

[34] G. Altarelli and G. Parisi, Asymptotic freedom in parton language, Nucl. Phys. B 126 (1977) 298 [SPIRES].

[35] H. Kharraziha and L. Lönnblad, The linked dipole chain Monte Carlo, JHEP 03 (1998) 006 [hep-ph/9709424] [SPIRES].

[36] H. Kharraziha and L. Lönnblad, LDCMC version 1.0 program and manual, Comput. Phys. Commun. 123 (1999) 153.

[37] G. Gustafson, Dual description of a confined color field, Phys. Lett. B 175 (1986) 453 [SPIRES].

[38] G. Gustafson and U. Pettersson, Dipole formulation of QCD cascades, Nucl. Phys. B 306 (1988) 746 [SPIRES].

[39] L. Lönnblad, ARIADNE version 4: a program for simulation of QCD cascades implementing the color dipole model, Comput. Phys. Commun. 71 (1992) 15 [SPIRES].

[40] B. Andersson, G. Gustafson and B. Söderberg, A general model for jet fragmentation, Z. Phys. C 20 (1983) 317 [SPIRES].

[41] B. Andersson, G. Gustafson, G. Ingelman and T. Sjöstrand, Parton fragmentation and string dynamics, Phys. Rept. 97 (1983) 31 [SPIRES].

[42] T. Sjöstrand, S. Mrenna and P.Z. Skands, A brief introduction to PYTHIA 8.1, Comput. Phys. Commun. 178 (2008) 852 [arXiv:0710.3820] [SPIRES].

[43] T. Sjöstrand, S. Mrenna and P.Z. Skands, PYTHIA 6.4 physics and manual, JHEP 05 (2006) 026 [hep-ph/0603175] [SPIRES].

[44] UA4 collaboration, D. Bernard, 1 et al., Large t elastic scattering at the CERN SPS collider at $\sqrt{s}=630 \mathrm{GeV}$, Phys. Lett. B 171 (1986) 142 [SPIRES].

[45] NA22 collaboration, M. Adamus et al., Study of elastic $\pi^{+} p, K^{+} p$ and pp scattering at $250 \mathrm{GeV} /$ c, Phys. Lett. B 186 (1987) 223 [SPIRES].

[46] CDF collaboration, F. Abe et al., Measurement of small angle anti-proton-proton elastic scattering at $\sqrt{s}=546 \mathrm{GeV}$ and $1800 \mathrm{GeV}$, Phys. Rev. D 50 (1994) 5518 [SPIRES]. 
[47] CDF collaboration, F. Abe et al., Measurement of the anti-proton proton total cross-section at $\sqrt{s}=546 \mathrm{GeV}$ and $1800 \mathrm{GeV}$, Phys. Rev. D 50 (1994) 5550 [SPIRES].

[48] E-710 collaboration, N.A. Amos et al., A luminosity independent measurement of the $\bar{p} p$ total cross-section at $\sqrt{s}=1.8$ TeV, Phys. Lett. B 243 (1990) 158 [SPIRES].

[49] E-710 collaboration, N.A. Amos et al., Anti-proton-proton elastic scattering at $\sqrt{s}=1.8 \mathrm{TeV}$ from $|t|=0.034 \mathrm{GeV} / \mathrm{c}^{2}$ to $0.65 \mathrm{GeV} / \mathrm{c}^{2}$, Phys. Lett. B 247 (1990) 127 [SPIRES].

[50] E-811 collaboration, C. Avila et al., The ratio, $\rho$, of the real to the imaginary part of the $\bar{p} p$ forward elastic scattering amplitude at $\sqrt{s}=1.8$ TeV, Phys. Lett. B 537 (2002) 41 [SPIRES].

[51] UA4/2 collaboration, C. Augier et al., Measurement of the proton-anti-proton total cross-section at the $S \bar{p} p S$ collider by a luminosity dependent method, Phys. Lett. B 344 (1995) 451 [SPIRES].

[52] M.M. Block, F. Halzen and T. Stanev, Extending the frontiers: reconciling accelerator and cosmic ray pp cross sections, Phys. Rev. D 62 (2000) 077501 [hep-ph/0004232] [SPIRES].

[53] ZEUS collaboration, S. Chekanov et al., Study of deep inelastic inclusive and diffractive scattering with the ZEUS forward plug calorimeter, Nucl. Phys. B 713 (2005) 3 [hep-ex/0501060] [SPIRES].

[54] H1 collaboration, A. Aktas et al., Measurement of deeply virtual Compton scattering at HERA, Eur. Phys. J. C 44 (2005) 1 [hep-ex/0505061] [SPIRES].

[55] H1 collaboration, F.D. Aaron et al., Measurement of deeply virtual compton scattering and its t-dependence at HERA, Phys. Lett. B 659 (2008) 796 [arXiv:0709.4114] [SPIRES].

[56] L. Lönnblad, ThePEG, PYTHIA7, HERWIG++ and Ariadne, Nucl. Instrum. Meth. A 559 (2006) 246 [SPIRES].

[57] M. Bähr et al., HERWIG++ physics and manual, Eur. Phys. J. C 58 (2008) 639 [arXiv:0803.0883] [SPIRES].

[58] A. Buckley et al., Rivet user manual, arXiv:1003.0694 [SPIRES].

[59] ATLAS collaboration, G. Aad et al., Measurement of underlying event characteristics using charged particles in pp collisions at $\sqrt{s}=900 \mathrm{GeV}$ and 7 TeV with the ATLAS detector, Phys. Rev. D 83 (2011) 112001 [arXiv:1012.0791] [SPIRES].

[60] ATLAS collaboration, G. Aad et al., Charged-particle multiplicities in pp interactions measured with the ATLAS detector at the LHC, New J. Phys. 13 (2011) 053033 [arXiv:1012.5104] [SPIRES].

[61] CDF collaboration, T. Affolder et al., Charged jet evolution and the underlying event in proton-anti-proton collisions at 1.8 TeV, Phys. Rev. D 65 (2002) 092002 [SPIRES].

[62] A. Buckley et al., General-purpose event generators for LHC physics, Phys. Rept. 504 (2011) 145 [arXiv:1101.2599] [SPIRES].

[63] R. Corke and T. Sjöstrand, Interleaved parton showers and tuning prospects, JHEP 03 (2011) 032 [arXiv:1011.1759] [SPIRES].

[64] CDF collaboration, R. Field, Min-bias and the underlying event in Run 2 at CDF, Acta Phys. Polon. B 36 (2005) 167 [SPIRES].

[65] C. Flensburg, G. Gustafson, L. Lönnblad and A. Ster, Correlations in double parton distributions at small $x$, JHEP 06 (2011) 066 [arXiv:1103.4320] [SPIRES]. 